\title{
Analysis of miRNA-mediated Regulation of Flowering Induction in Lilium $\times$ formolongi
}

\author{
Qian Zhang \\ Beijing Forestry University \\ Yu-Qian Zhao \\ Beijing Forestry University \\ Xue Gao \\ Beijing Forestry University \\ Guixia Jia ( $\nabla$ gxjia@bjfu.edu.cn ) \\ Beijing Forestry University
}

\section{Research article}

Keywords: Lilium × formolongi, development, flowering, miRNA

Posted Date: December 1st, 2020

DOI: https://doi.org/10.21203/rs.3.rs-113690/v1

License: () (1) This work is licensed under a Creative Commons Attribution 4.0 International License. Read Full License

Version of Record: A version of this preprint was published at BMC Plant Biology on April 20th, 2021. See the published version at https://doi.org/10.1186/s12870-021-02961-3. 


\section{Abstract}

Background: MicroRNAs play pivotal roles in plant vegetative phase change and flowering induction via integrating into multiple flowering pathways. Lilium $\times$ formolongi is an important ornamental lily cultivar that can flower within one year after sowing. However, it remains unresolved how miRNA-mediated regulation networks contribute to the $L . \times$ formolongi characteristics of a short vegetative growth period and rapid flowering.

Results: In this study, five small RNA libraries and one degradome library were constructed for $L . \times$ formolongi during vegetative growth and flowering initiation, and 366 conserved miRNAs and 32 novel miRNAs were identified. Additionally, 84 miRNAs were significantly differentially expressed during development. A total of 396 targets of 185 miRNAs were identified and validated through degradome sequencing. Gene Ontology (GO) and Kyoto Encyclopedia of Genes and Genomes (KEGG) pathway analyses showed that functions of the targets were top enriched in the cold and cadmium ion responses, pentose phosphate pathway and carbon fixation in photosynthetic organisms pathway. Furthermore, among 23 differentially expressed miRNAtarget pairs, the miR156s-LfSPL2, miR172a-LfAP2 and miR164a- LfNAC pairs as well as miR159a-LfSPL2 were found to be relevant to flowering based on the correlation analysis of expression profiles in the miRNA libraries, degradome and transcriptome. A coexpression regulatory network focused on differentially expressed pairs was also constructed by WGCNA, and 14 miRNAs were considered putative key miRNAs during vegetative development and flowering induction. miR156a/ d/ e showed particularly strong relationships with other miRNAs in the coexpression network.

Conclusions: This study provides cues for the further exploration of the regulatory mechanisms of short vegetative development and flowering in $L$. $\times$ formolongi.

\section{Background}

Lilium (Liliaceae family) is an important genus including crops that are valued for their ornamental characteristics, edible bulbs and medical use. They can be propagated by either sexual or asexual propagation. Sexual propagation through seed reproduction enables breeders to obtain virus-free seedlings and maintain abundant genetic diversity [1]. In most lily cultivars and wild species, at least 1 year of vegetative growth is required for flowering after sowing [2]. However, L. × formolongi, a popular cut flower cultivar with the characteristic of a shortened vegetative phase, can undergo floral transition within one year after germination [3]. Therefore, the investigation of molecular pathways involved in $L$. $\times$ formolongi vegetative development and flowering initiation is necessary to shorten the vegetative growth period and advance the flowering time of lily.

Vegetative growth after germination is necessary for angiosperm plants to achieve competence to flower and respond to extraneous stimuli after the juvenileadult stage transition, followed by the transition to reproductive growth [4]. As such, the optimal timing of the transition from the vegetative to the reproductive phase and flowering is precisely controlled by a complex genetic network composed of multiple pathways. In the annual plant Arabidopsis thaliana, external factors such as day length and temperature affect the photoperiod and vernalization pathways. They control phase transitions and flowering time in coordination with ageing, gibberellic acid (GA), trehalose 6-phosphate (T6P) and autonomous pathways governed by endogenous factors including age, hormones and carbohydrate status $[5,6]$. Currently, a large number of studies suggest that miRNAs are also integrated into flowering pathways, in which they perform orchestrated functions.

Mature plant miRNAs exert regulatory effects primarily by directly cleaving mRNAs or repressing the translation of specific mRNAs [7, 8]. In Arabidopsis, several endogenous conserved miRNAs and their targets are integrated into the ageing pathway, in which they influence development phase changes and flowering time $[9,10]$. miR156, the orchestrator of the pathway, plays a master regulatory role in vegetative development via transcript cleavage or the translational repression of target SQUAMOSA PROMOTER BINDING PROTEIN-LIKE (SPL/ SBP) genes, resulting in the transition to the adult stage [11, 12]. Conversely, miR172 targets six APETALA 2 (AP2)-like genes that function in flowering repression, and the abundance of miR172 increases with age [13, 14]. In apices, miR172 acts downstream of the miR156/ SPL module, and SPL9 and SPL 15 can alter the expression of miR172 by directly binding to the miR172 precursor MIR172b [15]. In addition to the two components of the ageing pathway, the examination of the phenotypes of mutant and transgenic plants has demonstrated that miR159, which is repressed by the DELLA protein in the GA pathway, is involved in the vegetative phase transition, independent of GA, and represses the expression of miR156 [16, 17]. In tomato, miR156-SISBPs interact with GA and the miR319-LANCEOLATE $(L A)$ module to affect flowering induction and the floral transition [18]. Additionally, overexpressed miR171 can activate miR156 to influence the vegetative-to-reproductive phase change in barley [19].

Many species-specific or less conserved miRNAs have also been identified and further confirmed to be involved in flowering induction in plants. Monocotspecific miRNA miR528 has been found to promote rice flowering induction under long-day conditions [20]. Brassicaceae-specific miRNA miR824 and its target AGL 16 modulate flowering time by repressing the expression of FT under long-day conditions in Arabidopsis [21]. Pooideae-specific miR5200 has been shown to regulate the photoperiod-mediated flowering transition by guiding the sequence-specific cleavage of $F T$ orthologues in Pooideae plants [22].

As a geophyte with underground storage organs, previous studies have confirmed that the flowering time of lily can be affected by multiple factors, including vernalization, photoperiod and bulb size $[23,24]$. However, the molecular regulatory factors underlying the vegetative phase transition and responses to various floral induction signals in lily are still unknown. On the other hand, the lily genome is large, complex and lacks a reference genome sequence [25]. Nevertheless, sequencing technologies make it relatively easy to produce sufficient data for the identification of genes related to important agronomic traits, and this research could benefit from computational and degradome analyses that detect the targets of miRNAs rapidly and accurately [26, 27].

Therefore, five miRNA libraries and one degradome library were constructed for $L$. $\times$ formolongi in key developmental stages. Databases, in conjunction with transcriptomic data, were further used to systematically identify and profile miRNAs and their targets during vegetative development and flowering initiation. Potential crucial flowering miRNA-target pairs were also identified. These results may contribute to elucidating the regulatory mechanism of miRNA-mediated short vegetative development in $L . \times$ formolongi and accelerating lily flowering. 


\section{Results}

\section{High-throughput sRNA sequencing in L. x formolongi}

To explore comprehensive miRNA data during L. × formolongi development, 5 small RNA libraries were constructed and sequenced from leaf and stem meristem tissues collected in 5 developmental stages (VJ_I, VJ_II, FI_I, FI_II and FD). In total, 87,720,826 raw reads were generated from these sRNA libraries (Table 1). After removing junk sequences, poor-quality tags and noncoding RNAs annotated in the Rfam database, approximately 9.9 million, 10.8 million, 14.7 million, 19.3 million and 5.6 million clean reads of $18-25 \mathrm{nt}$ in length that exactly matched the precursors deposited in miRBase 22.0 were obtained from the VJ_I, VJ_II, FI_I, FI_II and FD libraries, respectively. These miRNAs showed unique size distributions. The length distribution of $60,351,351$ valid reads and $14,126,406$ unique reads was centred on the $21-24 \mathrm{nt}$ range. The most frequent length of filtered reads among the five libraries was $24 \mathrm{nt}$, whereas it was $21 \mathrm{nt}$ for clean reads and $22 \mathrm{nt}$ for unique reads (Fig. 1a).

Table 1

High-throughput sequencing statistics of L. $\times$ formolongisRNAs

\begin{tabular}{|c|c|c|c|c|c|c|c|c|c|c|c|}
\hline \multirow{2}{*}{\multicolumn{2}{|c|}{ Category }} & \multicolumn{2}{|l|}{ VJ_I } & \multicolumn{2}{|l|}{ VJ_II } & \multicolumn{2}{|l|}{ FLII } & \multicolumn{2}{|l|}{ FI_II } & \multicolumn{2}{|l|}{ FD } \\
\hline & & Total & unique & Total & unique & Total & unique & Total & unique & Total & unique \\
\hline \multicolumn{2}{|c|}{ Raw reads } & $14,240,103$ & $4,165,620$ & $13,012,678$ & $5,070,705$ & $25,100,402$ & $4,417,437$ & $24,430,380$ & $5,493,407$ & $10,937,263$ & $2,363,859$ \\
\hline \multicolumn{2}{|c|}{$\begin{array}{l}\text { 3ADT \& length } \\
\text { filter }\end{array}$} & $3,253,881$ & $1,696,297$ & $1,726,013$ & $1,370,377$ & $8,960,668$ & $1,903,704$ & $4,503,056$ & $1,379,069$ & $5,065,486$ & 855,271 \\
\hline \multicolumn{2}{|c|}{ Junk reads } & 43,445 & 18,415 & 44,301 & 25,086 & $55,442 c$ & 15,965 & 59,104 & 21,952 & 31,103 & 10,933 \\
\hline \multirow[t]{6}{*}{ Rfam } & rRNA & 625,844 & 17,010 & 103,024 & 7,038 & 830,461 & 18,275 & 152,864 & 8,762 & 135,278 & 6,417 \\
\hline & tRNA & 334,519 & 3,726 & 271,546 & 3,448 & 429,462 & 3,870 & 364,860 & 3,249 & 74,368 & $1,669 \mathrm{c}$ \\
\hline & snoRNA & 4,261 & 380 & 5,215 & 436 & 20,391 & 926 & 19,562 & 890 & 17,995 & 843 \\
\hline & snRNA & 4,524 & 430 & 4,826 & 523 & 12,468 & 951 & 8,310 & 761 & 5,221 & 489 \\
\hline & others & 35,921 & 1,242 & 8,421 & 678 & 97,281 & 1,808 & 11,959 & 1,010 & 14,357 & 683 \\
\hline & Total & $1,005,069$ & 22,788 & 393,032 & 12,123 & $1,390,063$ & 25,830 & 557,554 & 14,672 & 247,219 & 10,101 \\
\hline \multicolumn{2}{|c|}{ Repeats } & 8,622 & 451 & 8,427 & 458 & 19,758 & 680 & 10,301 & 708 & 3,934 & 323 \\
\hline \multicolumn{2}{|c|}{ valid reads } & $9,932,250$ & $2,427,801$ & $10,843,854$ & $3,662,791$ & $14,681,011$ & $2,471,404$ & $19,303,311$ & $4,077,107$ & $5,590,925$ & $1,487,303$ \\
\hline
\end{tabular}

\section{Identification of conserved miRNAs in L. $\times$ formolongi}

The valid reads from the five libraries were subjected to mapping with the precursors deposited in miRBase 22.1 and the transcriptome of $L$. $\times$ formolongi to identify conserved miRNAs. A total of 345 conserved miRNA precursors and 366 unique miRNAs belonging to 116 miRNA families were discovered, and the genomic localization was obtained for a majority of these miRNAs (Fig. 1b, Additional file 1: Table S1). In addition, the numbers of homologs were counted and varied among the different miRNA families. The largest family was the miR156 family, with 22 members, followed by the miR166 and miR169 families, which had 17 and 14 members, respectively. However, miR162, miR391, miR828 and miR5072 were each represented by only one member. Furthermore, two miR2916 members, one miR5523 member and one miR11602 member were identified as known miRNAs (group 1) whose reads and precursors could be perfectly mapped to the reported Lilium miRNAs/ pre-miRNAs sequences in miRBase and the transcriptome.

The relative abundance of these conserved miRNAs during lily development was characterized. As shown in Fig. 1c, the miRNAs exhibited remarkably different levels of abundance. The top read counts of miRNAs were obtained for the miR166 family, followed by the miR159 and miR408 families during developmental stages.

\section{Identification of novel miRANs in L. × formolongi}

The unannotated reads that were mapped to the transcriptome of $L . \times$ formolongi were filtered for novel miRNA prediction according to the criteria for the hairpin structure of the precursor. Collectively, 32 novel miRNAs were found among the five miRNA libraries (Additional file 1: Table S2). The lengths of these mature novel miRNAs and the hairpin lengths of the novel pre-miRNAs were between 19 and $25 \mathrm{nt}$ and 55 and 191 nt, respectively. The majority of the miRNAs in these intervals were $21 \mathrm{nt}$ and $164 \mathrm{nt}$ long, respectively. The free energy of these secondary structures required to maintain stable hairpin structures ranged from -125.5 to $-20.9 \mathrm{kCal} / \mathrm{mol}$. Precursors forming stem loop structures among the novel miRNAs, such as miRn5, miRn8 and miRn10, were predicted and are presented in Additional file 2: Fig. S1.

\section{Differentially expressed miRNAs during development}


To identify the key miRNAs playing regulatory roles during vegetative growth and flowering initiation in lily, 75 conserved miRNAs and 9 novel miRNAs were identified as showing significant differences in expression (Additional file 1: Table S3). Cluster analysis of their expression patterns was performed, and a heat map showing their distinct expression profiles was generated (Fig. 2). These miRNAs were characterized by three expression profiles with different expression changes from the VJ_I to FI_Il stages before FD.

The majority of miRNAs, including 50 conserved miRNAs and 9 novel miRNAs, presented high expression levels in the VJ (VJ_I and VJ_II) stage and were downregulated during later stages of development. In the miR156 family, most miR156s displayed consistently decreased expression, with the highest expression levels in the VJ_I or VJ_Il stage. Similar to miR156s, most miR166 families exhibited expression peaks in the VJ_ll stage. By contrast, 20 conserved miRNAs exhibited elevated expression from the VJ to FI_Il stage or FD stage. miR159d, miR159f, miR172a and miR171 homologs exhibited upregulated expression levels from VJ_I to FI_Il, and several less conserved miRNAs, such as miR5540, miR8708 and miR3630a/ b, maintained increased expression from the VJ to FD stages. In addition, many differentially expressed miRNAs with fluctuating transcription levels were found in the libraries. The expression of miR156k, miR2592bj and miR167e/ a was downregulated in the VJ_Il and FI_Il stages.

\section{Degradome sequencing}

By combining the results of degradome and computational target prediction for the annotated corresponding mRNAs, 396 target transcripts of 235 miRNAs were globally identified in the degradome and analysed by detecting mRNA fragments that were diagnostic of miRNA-directed cleavage, including 157 conserved miRNAs (Fig. 3, Additional file 1: Table S4) and 28 novel miRNAs (Additional file 1: Table S5). By distinguishing occupied transcript positions on the basis of abundance in the degradome, we classified the identified targets into 5 categories according to the criteria indicated in previous studies [28]. Among these identified targets, $24,7,179,25$ and 184 belonged to categories $0,1,2,3$ and 4 , respectively.

In total, 6473 of the target sites were confirmed by degradome sequencing, and 4076 targets of 367 miRNA reads were simultaneously detected by computational target prediction and experimental sequencing. The enriched $\mathrm{GO}$ items were analysed to elucidate the functions of the identified targeted genes. Among biological processes, 'oxidation-reduction process' (GO: 0055114), 'response to cold' (GO: 0009409) and 'response to cadmium ion' (GO: 0046686) were the most abundant categories. 'Nucleus' (GO: 0005634) and 'ATP binding' (GO: 0005524) were the most highly represented cellular component and molecular function categories, respectively (Fig. 4a). The enriched KEGG pathway analyses showed that the pentose phosphate pathway (ko00030), carbon fixation in photosynthetic organisms (ko00710) and flavonoid biosynthesis (ko00941) were the most enriched pathways (Fig. 4b).

According to the functional annotations, putative flowering-related targets were identified. They consisted of squamosal promoter-binding, floral homeotic protein APETELA2-like, MADS-box, NAC domain-containing, R2R3-MYB, GAMYB and hormone signalling auxin response factor 18-like proteins (Table 2). We also found that the functions of $\angle F S P 2$ and $L F N A M$, targeted by miR156s and miR164a, were enriched in starch and sucrose metabolism, pentose and glucuronate interconversion pathways. Furthermore, both miR156s and miR159a were indicated to regulate the common target $L F S P L 2$ according to the identification of different target sites (Fig. 5a). miR159a and miR319f targeted LfGAMYB sharing a common targeting site (Fig. 5b). These transcripts targeted by miRNAs were considered candidate key regulators during vegetative development and flowering induction in lily.

Table 2

Identification of putative flowering-related target transcripts in L. $\times$ formolongi

\begin{tabular}{|c|c|c|c|c|}
\hline miR_name & Transcript & Transcript Annotation & Symbol & Term \\
\hline $\begin{array}{l}\text { Ifo- } \\
\text { miR156a,k,e,ac,d,g,y,r,h }\end{array}$ & Unigene18229_All & $\begin{array}{l}\text { squamosa promoter-binding-like } \\
\text { protein } 12 \text {-like }\end{array}$ & LfSPL2 & Starch and sucrose metabolism- \\
\hline $\begin{array}{l}\text { Ifo- } \\
\text { miR156a,e,ac,d,g,r,y }\end{array}$ & Unigene9969_All & predicted protein & LfSPL3 & - \\
\hline Ifo-miR159a,f,c & Unigene2305_All & transcription factor GAMYB & $\angle f G A M Y B$ & developmental process \\
\hline Ifo-miR159a & Unigene18229_All & $\begin{array}{l}\text { squamosa promoter-binding-like } \\
\text { protein } 12 \text {-like }\end{array}$ & LfSPL2 & Starch and sucrose metabolism- \\
\hline Ifo-miR159e & CL730.Contig4_All & transcription factor TCP2-like & & - \\
\hline Ifo-miR160a,f,g,b,d & CL6847.Contig1_All & auxin response factor 18 -like & LfARF & root cap development; auxin mediated signaling \\
\hline \multirow[t]{2}{*}{ Ifo-miR164a } & CL6145.Contig1_All & NAC domain-containing protein & LfNAC & - \\
\hline & Unigene9281_All & $\begin{array}{l}\text { no apical meristem-related } \\
\text { protein }\end{array}$ & LfNAM & $\begin{array}{l}\text { Starch and sucrose metabolism; Pentose and } \\
\text { glucuronate interconversions; }\end{array}$ \\
\hline \multirow[t]{2}{*}{ Ifo-miR172a } & CL1418.Contig1_All & $\begin{array}{l}\text { vesicle-associated membrane } \\
\text { protein }\end{array}$ & & response to salt stress \\
\hline & Unigene18548_All & $\begin{array}{l}\text { floral homeotic protein APETALA } \\
\text { 2-like }\end{array}$ & $\angle f A P 2$ & - \\
\hline Ifo-miR319f & Unigene2305_All & transcription factor GAMYB & $\angle f G A M Y B$ & developmental process \\
\hline Ifo-miR394 & Unigene14281_All & F-box family protein & & regulation of auxin mediated signaling \\
\hline Ifo-miR828 & Unigene3673_All & transcription factor R2R3-MYB & & \\
\hline Ifo-miR5179 & Unigene7245_All & MADS-box transcription factor & LfAGL 15 & regulation of transcription, DNA-dependent \\
\hline
\end{tabular}




\section{Correlation analysis of miRNA expression profiles and miRNA target genes}

To investigate the crucial participants and their variable trends during lily vegetative development and flowering initiation, the 28 detected target transcripts of significantly differentially expressed miRNAs obtained from the degradome were applied for the analysis of differentially expressed genes (DEGs). There were 15 DEGs corresponding to 23 differentially expressed miRNAs in the transcriptome. A heatmap was generated to show the reverse expression profiles of 23 miRNA-target pairs in the transcriptome and miRNA libraries from the VJ (vegetative growth) to FD stages (Fig. 6). Among these pairs, the members of the miR156 family were most highly expressed and displayed significantly downregulated expression levels, while the expression of their target $L F S P L 2$ was increased during development. Putative flowering-related miRNA-target pairs such as miR172a-LfAP2, miR156s/ miR159a-LfSPL2 and miR164a- $f$ fNAC were also identified in the correlation analysis.

Based on the results of the correlation analysis and the functional analysis of targets, 12 miRNA-target pairs that showed distinct expression patterns and may take part in flowering induction were selected to verify the accuracy of the sequencing results by performing qRT-PCR analysis. As depicted in Fig. 7a-j, these 10 candidate miRNAs were indicated to show substantially similar expression changes to those indicated by the data from miRNA libraries. A portion of these miRNAs were inversely correlated with their corresponding targets, such as miR156a-LfSPL2/ LfSPL3, miR159a-LfGAMYB, miR397a-LfLACs, miR160aLFARF18, miR5139-LFFAO2, and miR5179-LfAGL15, which confirmed the negative relationship in the expression profile of miRNA-mRNA modules. Moreover, the expression profiles of the miRNAs from the VJ_I to FD stages were diverse. miR160a was gradually upregulated after the VJ_I stage, while four miRNAs, including miR159a, miR172a, miR167a and miR171b, exhibited upregulated expression in the FI_I stage. Both miR5139b and miR5179 presented expression peaks in the VJ_Il stage. Moreover, all of these miRNAs exhibited a remarkable upregulation of transcript abundance from the FD to VB stages, with the exception of miR159f, which exhibited decreased expression at the FD stage.

In addition, we assayed the tissue-specific expression profiles of two significantly differentially expressed miRNAs, miR159a and miR160a (Fig. 7k, I). They showed similar expression patterns in different organs and tissues from the VJ to VB stages. Both presented high expression in roots and stems in the flowering induction (FI_I, FI_II) stages. Expression peaks of miR159a and miR160a were found in leaves at the FI_I stage. However, the level of miR159a increased from the VJ to FD stages in the apical meristem and bulb scales. In visible floral buds, the strikingly highest expression of mR159a was observed. Likewise, miR160a showed the highest expression level at the FD stage in the apical meristem.

\section{Gene coexpression network analysis}

To mine the highly correlated miRNA clusters that were relevant to flowering induction, we used miRNA data obtained from high-throughput sequencing to conduct correlation network analysis by WGCNA. After testing for abnormal samples or genes, correlation networks were constructed by setting the soft threshold to 12 (Additional file 4: Fig. S3-6). As a result, 5 miRNA coexpression modules comprising 70 (blue module), 40 (brown module), 53 (green module), 82 (turquoise module) and 33 (yellow module) interconnected miRNAs were found, as shown in Additional file 1: Table S7. We also recognized 14 miRNAs belonging to differentially expressed miRNA-target pairs in the blue module (miR169b, miR156a/ h/ e/ y), brown module (miR164a, miR172a, miR2592bj), green module (miR160a), turquoise module (miR156d/ g/ ac) and yellow module (miR398a, miR397a). The greatest number of these miRNAs belonging to differentially expressed pairs was found most in the blue module; in this regard, the blue module could be considered the key module related to critical stages of vegetative development and flowering initiation in lily.

We also extracted a subnetwork containing 795 edges connected with the differentially expressed miRNAs, which also included their targets (Additional file 1: Table S8). In the regulatory network, miR156a/ d/ e exhibited the greatest numbers of interactions with other sequences, suggesting their core roles among the coexpressed genes (Fig. 8). Moreover, phylogenetic analysis demonstrated that miR156s were most closely related to Arabidopsis. They are likely candidate miRNAs involved in lily flowering induction, and the specific roles of the members of this family require further validation and investigation (Additional file 3 :

Fig. S2).

\section{Discussion}

\section{Potential crucial miRNAs in vegetative development and flowering induction}

The role of miRNAs in flowering induction has been confirmed in different plants, such as Arabidopsis, rice, maize, apple and tomato [18, 29, 30]. The mechanism of miRNA functions in the genetic network underlying lily vegetative development and flowering initiation is poorly understood.

In our study, 84 miRNAs showing significant differential expression were identified, and the analysis of their expression dynamics during lily development was conducted. The greatest numbers of differentially expressed miRNAs were identified and strongly expressed in the VJ_I and VJ_II stages among the small RNA libraries. These results were consistent with our hypothesis, and we reason that miRNA-mediated modulation is crucial for vegetative development by integrating environmental and endogenous factors to prepare for the induction of flowering.

In the L. $\times$ formolongi libraries, miRNAs belonging to the miR156 family were the most frequent among the differentially expressed miRNAs. In Arabidopsis, miR156 is an important orchestrator of the ageing pathway that controls the vegetative-to-reproductive transition and shows very high expression in the vegetative phase [31]. The conserved expression profile of miR156 members has been confirmed in many plants, such as rice [32], maize [33], tobacco [34] and the perennial woody species Acacia confuse, Eucalyptus globulus, and Quercus acutissima [35]. In L. × formolongi, the miR156 families shared similar expression profiles with those observed in other lily plants during development, demonstrating their evolutionary and functional conservation and their

Page $5 / 24$ 
dominant role during vegetative development. Nevertheless, the expression level of miR156k fluctuated and was upregulated in the FI_I stage. In Arabidopsis and rice, Ath-MIR156A/ C and OsmiR156b/ $h$ play master roles among miR156 precursors [36-38]. We observed that the precursors of miR156s (MIR156k) were phylogenetically closely related to Arabidopsis sequences and that MiR156k exhibited the closest relationships with Ath-MIR156A/ C, which clustered into the same branch (Additional file 3: Fig. S2), indicating that miR156k precursors may have a greater effect on the abundance of miR156 during lily flowering induction than during the juvenile-adult phase transition.

A prior study in Arabidopsis confirmed that another crucial regulator, miR172, exhibits an inverse expression pattern with miR156 and shows consistently upregulated expression during development to regulate the phase transition as well as floral organ identity [39, 40]. There are five miR172 family members and miR172b could be negatively regulated by miR156e to influence the juvenile-adult phase change [31]. In the perennial species Jatropha curcas, only two miR172s have been identified [41]. However, ten miR172s and only one miR172 (miR172a) were identified among the differentially expressed miRNAs in our libraries. The expression of miR172a was elevated from the VJ to FI_Il stages, followed by a decline in the FD stage. Hence, we consider miR172a to exhibit relatively complex or species-specific functions in lily flowering induction or other aspects of development.

In Arabidopsis and rice, miR159a, miR167a and miR160a affect the development of anthers and sepals [42-44]. These highly conserved miRNAs also showed significantly upregulated expression in the VB stage, suggesting their important roles in floral organ formation during early floral development in lily. In addition, miR5179 affects the diversification of perianth organs by cleaving MADS-box genes in orchids [45]. In contrast, miR5179 presented upregulated expression in the VB stage but exhibited strikingly higher expression in the VJ stage, indicating that this less conserved miRNA, which is rarely observed in other plants, may exhibit a more crucial function during the vegetative development of lily than during flower development.

\section{Flowering-related miRNA-mRNA modules in L. x formolongi}

miRNAs carry out their biological functions by regulating the expression of specific mRNAs. We identified targets that encoded flowering-related transcription factors or proteins. SPL genes are targeted by miR156 and regulate many aspects of plant vegetative development as important components of multiple pathways [46]. In Arabidopsis, $10 S P L$ genes are regulated by miR156, and AtSPL3/ 14/ 15/ 9 can promote flowering [4, 47]. SPL7 and SPL8 participate in flowering modulation and are targeted by miR156 in grasses [48]. Among the 11 LfSPL members, two homologs, $L f S P L 2$ and $L f S P L 3$, were targeted by miR156s in L. $\times$ formolongi, and only miR156-LfSPL2 pairs were differentially expressed. Moreover, miR156a/ d/ e were located at the cores of the coexpression networks. Accordingly, miR156a/ d/ e-LfSPL2 modules may be the most critical regulators of the ageing pathway during vegetative development and flowering induction. The mechanism by which the miR156-LfSPL module is involved in the genetic network underlying flowering requires further intensive investigation and verification.

Previous studies have confirmed that miR159 regulates the juvenile-to-adult phase transition through a canonical miR159-GAMYB/ MYB33 interaction and the repression of miR156 as well as the target $S P L 9$ in Arabidopsis $[16,49]$. Interestingly, we found that miR159a regulated $L F S P L 2$ simultaneously with miR156s, in addition to $\angle F G A M Y B$, in this study. The expression level of $L F S P L 2$ was not negatively correlated with miR159a from the VJ to FI_I stages, and miR159a exhibited a high expression level in the FI_I stage. Hence, the results imply that miR159a might be involved in a new flowering pathway through which it affects vegetative development and flowering initiation by regulating the $S P L$ gene directly, and this regulatory module is worthy of validation in the future.

\section{Crucial factors affecting the vegetative development of lily}

A recent study conducted in our laboratory suggested that when the seedlings of $L . \times$ formolongi undergo bolting, the seedlings transition into the reproductive stage, and the juvenile-adult transition is accomplished [3]. Therefore, we focused on the miRNA-mediated regulatory mechanism during flowering induction, particularly during vegetative development in the rosette stage of $L . \times$ formolongi, and the factors affecting this process.

In the molecular regulatory network, sugar acts upstream of miR156 to alter miR156 expression [36]. T6P influences flowering in the leaves and apical meristem by regulating FT as a representative of carbohydrate conditions [6]. Previous studies have shown that daylength strongly affects the bolting time and quality of $L . \times$ formolongi [24]. Temperature also plays an important role in vegetative development. Cold exposure induces flowering by breaking bulb dormancy and influences the growth of bulbous plant species [50]. Our study showed that the functions of the identified targets were enriched in cadmium ion, cold response and sugar metabolism pathways, including the pentose phosphate pathway and carbon fixation in photosynthetic organisms. Thus, vegetative development may be closely associated with carbohydrates, cold or photoperiod in $L$. × formolongi via miRNAs. LfSPL2 and LfNAM, which are targeted by miR156s and miR164a, respectively, were identified in the sugar metabolism pathway. Hence, the impacts of these factors on the vegetative development of $L$. $\times$ formolongi through the potential miRNA-mRNA modules is also an important topic awaiting future exploration.

\section{Conclusions}

To our knowledge, the present study provides the first integrated transcriptomic, miRNA library and degradome analysis conducted to obtain comprehensive knowledge of miRNAs and their targets during flowering initiation in lily. Crucial miRNAs and miRNA-target pairs involved in vegetative development and floral induction in L.x formolongi were identified via the correlation analysis of sequencing datasets. Factors including carbohydrates, cold and photoperiod and associated enriched target functions may affect vegetative development and flowering induction. These results will help us to gain broader knowledge of the miRNA-mediated mechanism modulating L.x formolongi vegetative development and flowering and will be of great importance for regulating growth and development processes in lily.

\section{Methods}




\section{Plant Materials and Samples Collection}

The seeds of L. $\times$ formolongi ('Razan No. 2') (purchased from Murakami Seed, Ibaraki, Japan) were sown after stratification at $4^{\circ} \mathrm{C}$ for one month. Seedlings with 2-3 rosette leaves were transplanted into individual pots and grown in a chamber under a $16 \mathrm{~h} \mathrm{light}\left(25^{\circ} \mathrm{C}\right) / 8 \mathrm{~h}$ dark cycle $\left(16^{\circ} \mathrm{C}\right)$ with $70 \%$ humidity, illuminated by white light with a $320 \mu \mathrm{mol} \cdot \mathrm{m}^{-2} \cdot \mathrm{s}^{-1}$ photosynthetic photon flux density.

A previous study in our laboratory confirmed the key characteristics of vegetative development and flowering initiation in L. $\times$ formolongi under long-day conditions through morphological and gene expression pattern investigations [3]. Therefore, fresh leaves and apical stems were collected for small RNA library construction during different developmental stages, including the 2-3 rosette leaf stage (vegetative juvenile_l, VJ_I), 5-6 rosette leaf stage (vegetative juvenile_II, VJ_II), 1-2 internode stage (flowering induction_I, FI_I), 5-6 internode stage (flowering induction_II, FI_II) and 9-10 internode stage (floral differentiation, FD). Similar samples from these stages together with fresh leaves and buds collected from 70-71 internodes (visible floral bud stage, VB) were prepared for qRT-PCR analysis. To examine the tissue-specific expression patterns of differentially expressed miRNAs, fresh leaves, apical meristems, middle stems, bulb scales (in the middle of the bulb), roots and tiny floral bubs (approximately $2 \mathrm{~cm}$ ) were also collected from one plant from the VJ_I to VB stages. The samples obtained from three individual plants were immediately frozen in liquid nitrogen, followed by storage at $-80^{\circ} \mathrm{C}$ for further study.

\section{Small RNA library construction and sequencing}

Total RNA was extracted using the EASY spin Plus Plant RNA kit. After ligating the RNA with 3' and 5' adapters, small RNAs were used for reverse transcription and amplification, and gel-purified small RNA libraries of $L$. $\times$ formolongi at different development and flowering initiation stages were constructed with Illumina HiSeq 2000/2500 technology (LC Bio, Hangzhou, China). Each reaction was performed in triplicate.

\section{Bioinformatic analysis of sRNA data}

To obtain clean reads, sequence adapters, junk sequences, and low-quality tags were filtered out using an in-house program, ACGT101-miR (LC Sciences, Houston, Texas, USA). Subsequently, the quality reads that were aligned to the mRNA (http://www.icrisat.org/gt-bt/ICGGC/GenomeSequencing.htm), RFam (http://rfam.xfam.org) and Repbase (http://www.girinst.org/education/index.html) databases were discarded. The remaining valid reads of 18-25 nt were further processed to summarize the length distribution, and mapping to specific species precursors in miRBase 22.0 via BLAST searches and transcriptome analysis of $L . \times$ formolongi were performed to identify known miRNAs. According to whether the reads and precursors could be matched to sequences in miRBase and the transcriptome, they are categorized into 3 groups, including known miRNAs (perfectly matched sequences, group 1 ) and conserved miRNAs (group 2).

After extracting the known miRNAs, the valid reads that were unmapped in miRBase but were subjected to BLAST searches against the transcriptome of $L$. $\times$ formolongi were selected for novel miRNA identification. RNAfold software (http://rna.tbi.univie.ac.at/cgi-bin/RNAfold.cgi) was employed to predict the secondary structures of potential miRNA sequences. The detailed criteria applied were as follows: (1) numbers of nucleotides in a single bulge in the stem and the mature region were $\leq 12$ and 4 , respectively; (2) in a predicted hairpin, number of base pairs in the stem region was $\geq 16$; (3) in the mature region, both numbers of biased errors and bulges in one bulge and the number of errors were $\leq 2$ [51].

\section{Analysis of Differentially Expressed miRNAs}

To identify the differentially expressed miRNAs in L. × formolongi during the flowering transition phase and development, the deep-sequencing counts were normalized, and differentially expressed miRNAs were identified by using the T-test as described in prior reports [52]. We calculated the expected Log2 (copy\#) values to indicate the original copy numbers of the samples. The T-test was used to obtain p-values via pairwise comparisons between libraries. The miRNAs with $p$-values $\leq 0.05$ in each test were defined as significantly differentially expressed miRNAs during lily development.

\section{Degradome library construction and target identification}

Total RNAs from the fresh leaves and apical meristem of $L . \times$ formolongi at five developmental stages were equally mixed as a single sample for constructing the degradome library according to published protocols [53]. Clean tags were generated and subjected to potential cleaved target identification by comparison with transcriptome sequences [54]. Thus, truncated transcripts resulting from endonucleolytic cleavage guided by miRNAs could be identified [55]. Furthermore, the alignment analysis of comparisons between identified miRNAs and mapped mRNAs was simultaneously performed using computational target prediction algorithms in TargetFinder (http://carringtonlab.org/resources/targetfinder) software to identify miRNA binding sites. Further annotation of targets was conducted in the Gene Ontology (GO) and Kyoto Encyclopedia of Genes and Genomes (KEGG) pathway databases for further miRNA-gene regulatory network analysis after all target unigenes were subjected to Blast2GO (http://www. blast2go.org/) analysis and aligned against the enriched KEGG pathways following a previously reported method [56].

\section{Quantitative real-time PCR (qRT-PCR) analysis}

Total RNA and miRNA were extracted from leaves and apical meristems at six developmental stages from the seedling to visible floral bud phases under longday conditions using RNA extraction kits (Aidlab Biotechnology Co., LTD., China; Tiangen Bio, Beijing, China) according to the manufacturer's instructions. 
Then, the obtained RNAs and miRNAs were subjected to quality characterization, poly (A)-enrichment and reverse transcription to obtain cDNA using a Prime Script Double Strand cDNA synthesis kit (Takara, Dalian, China) and Quant Script RT Kit (Tiangen Bio, Beijing, China). In addition, to assess tissue-specific miRNA expression, total miRNAs were separately extracted from different tissues from seedlings in the same developmental phases. qRT-PCR was carried out by using THUNDERBRIRD SYBR qPCR Mix Without Rox (Toyobo, Shanghai, China) on a Bio-Rad CFX96 system (CFX96 Touch, BIO-RAD, USA). Each reaction was performed in triplicate. The 5S RNA and EF genes were used as the internal reference genes for the normalization of the results. The primers for the miRNA and targets were designed using Beacon Designer 7 software, as indicated in Supplementary Table 6 . The $-2^{-\Delta \Delta C t}$ method was employed to statistically analyse these data [57].

\section{Coexpression network construction}

To generate a correlation network of miRNAs during lily development, an undirected weighted gene network was constructed with a weighted gene coexpression network analysis (WGCNA) algorithm. A tutorial for the WGCNA package in R is provided at https://horvath.genetics.ucla.edu/html/CoexpressionNetwork/Rpackages/WGCNA/Tutorials/index.html. The normalized miRNA expression data from the libraries were used for correlation network construction after gene screening with a power of 12 . We focused the analysis on module eigengenes whose targets were also significantly differentially expressed during lily flowering. The correlation network of interest was selected for visualization in Cytoscape (version 3.6.1). Moreover, the alignment of the sequences of miRNA precursors and the phylogenetic analysis of miR156 families were performed via the ClustalW and NJ methods in MEGA 6.06 software with default parameters. The precursor sequences of other species were obtained from miRBase, Release 21 (http://www.mirbase.org/index.shtml).

\section{Abbreviations}

miRNAs: MicroRNAs; FT: FLOWERING LOCUS T; T6P: Trehalose-6-phosphate; nt: Nucleotide; SPL: SQUAMOSA PROMOTER BINDING PROTEIN-LIKE; AP2: APETALA 2; GA: Gibberellin; ARF: AUXIN RESPONSE FACTORS, GO: Gene Ontology; KEGG: Kyoto Encyclopedia of Genes and Genomes; NJ: Neighbor-Joining; qRTPCR: Quantitative real time polymerase chain reaction; WGCNA: weighted gene coexpression network analysis; rRNA: Ribosomal RNA; tRNA: Transfer ribonucleic acid; snoRNA: Small nucleolar RNA; snRNA: Small nuclear RNA; sRNA: Small RNA; mRNA: Messenger RNA; NAC (NAM): NO APICAL MERISTEM; DEGs: Differentially expressed genes; LAC. LACCASE; FAO: FATTY ALCOHOL OXIDASE; AGL: AGAMOUS-LIKE

\section{Declarations}

\section{Ethics approval and consent to participate}

Not applicable.

\section{Consent for publication}

Not applicable.

\section{Avaliability of data and materials}

The dataset supporting the conclusions of this article is included within the article and its additional files

\section{Competing interests}

The authors declare that they have no competing interests.

\section{Funding}

This work was supported by National Key R\&D Program of China (Grant No. 2019YFD1000400) and National Natural Science Foundation of China (Grant No. 31772348). The funders did not have any role in the design of the study, collection, analysis, or interpretation of data or the writing of the manuscript.

\section{Authors' contributions}

GXJ designed the experiments. QZ and YQZ conducted the experiments. QZ performed the bioinformatics analysis with the help of XG and drafted the manuscript. GXJ directed and modified the manuscript. All authors read and approved the final manuscript.

\section{Acknowledgements}

Not applicable. 


\section{References}

1. Bakhshaie M, Khosravi S, Azadi P, Bagheri H, Tuyl JMV. Biotechnological advances in Lilium. Plant Cell Rep. 2016;35(9):1799-1826.

2. Fortanier EJ. Reviewing the length of the generation period and its shortening, particularly in tulips. Sci Hort. 1973;1(1):107-116.

3. Li YF, Zhang MF, Zhang M, Jia, GX. Analysis of global gene expression profiles during the flowering initiation process of Lilium $\times$ formolongi. Plant Mol Biol. 2017;94:361-379.

4. Huijser P, Schmid M. The control of developmental phase transitions in plants. Development. 2011;138(19):4117-4129.

5. Teotia S, Tang G. To bloom or not to bloom: role of microRNAs in plant flowering. Mol Plant. 2015;8(3):359-377.

6. Wahl V, Ponnu J, Schlereth A, Arrivault S, Langenecker T, Franke A, Feil R, Lunn JE, Stitt M, Schmid M. Regulation of flowering by trehalose-6-phosphate signaling in Arabidopsis thaliana. Science. 2013;339(6120):704-707.

7. Wilczynska A, Bushell M. The complexity of miRNA-mediated repression. Cell Death Differ. 2015;229(1):22-33.

8. Rogers K, Chen X. Biogenesis, turn over, and mode of action of plant microRNAs. Plant Cell. 2013;25:2383-2399.

9. Dario M, Griffithsjones S, Kim M. Small RNAs: Big impact on plant development. Trends Plant Sci. 2017;22(12):1056-1068.

10. Luo Y, Guo Z, Li L. Evolutionary conservation of microRNA regulatory programs in plant flower development. Dev Biol. 2013;380(2):133-144.

11. He J, Xu M, Willmann MR, McCormick K, Hu T, Yang L, Starker CG, Voytas DF, Meyers BC, Poethig RS. Threshold-dependent repression of SPL gene expression by miR156/miR157 controls vegetative phase change in Arabidopsis thaliana. PLoS Genet. 2018;14:e1007337.

12. Wang J, Czech B, Weigel D. MiR156- regulated SPL transcription factors define an endogenous flowering pathway in Arabidopsis thaliana. Cell. 2009;138(4):738-749.

13. Mathieu J, Yant LJ, Murdter F, Kuttner F, Schmid M. Repression of flowering by the miR172 target SMZ. PLoS Biol. 2009;7(7):e1000148.

14. Aukerman MJ, Sakai H. Regulation of flowering time and floral organ identity by a microRNA and its APETALA2-like target genes. Plant Cell. 2003;15(11):2730-2741.

15. Wu G, Park MY, Conway SR, Wang JW, Weigel D, Poethig RS. The sequential action of miR156 and miR172 regulates developmental timing in Arabidopsis. Cell. 2009;138(4):750-759.

16. Guo C, Xu Y, Shi M, Lai Y, Wu X, Wang H, Zhu Z, Poethig RS, Wu G. Repression of miR156 by miR159 regulates the timing of the juvenile-to-adult transition in Arabidopsis. Plant Cell. 2017;29:1293-1304.

17. Moon J, Suh SS, Lee H, Choi KR, Hong CB, Paek NC, Kim SG, Lee I. The SOC1 MADS-box gene integrates vernalization and gibberellin signals for flowering in Arabidopsis. Plant J. 2003;35:613-623.

18. Silva GF, Silva EM, Correa JP, Vicente MH, Jiang N, Notini MM, Junior AC, Jesus FA, Castilho P, Carrera E, Lopez-Dıaz I, Grotewold E, Peres LE, Nogueira FT. Tomato floral induction and flower development are orchestrated by the interplay between gibberellin and two unrelated microRNA-controlled modules. New Phytol. 2019;221:1328-1344.

19. Curaba J, Talbot M, Li Z, Helliwell C. Over-expression of microRNA171 affects phase transitions and floral meristem determinancy in barley. BMC Plant Biol. 2013;13:6.

20. Yang R, Li P, Mei H, Wang D, Sun J, Yang C, Hao L, Cao S, Chu C, Hu S, Song X, Cao X. Fine-tuning of miR528 accumulation modulates flowering time in rice. Mol Plant. 2019;12:1103-1113.

21. Hu J, Zhou Y, He F, Dong X, Liu L, Coupland G, Turck F, Meauxc J. miR824-regulated AGAMOUS-LIKE16 contributes to flowering time repression in Arabidopsis. Plant Cell. 2014;26:2024-2037.

22. Wu L, Liu D, Wu J, Zhang R, Qin Z, Liu D, Li A, Fu D, Zhai W, Mao L. Regulation of FLOWERING LOCUS T by a MicroRNA in Brachypodium distachyon. Plant Cell. 2013;25:4363-4377.

23. Lazare S, Zaccai M. Flowering pathway is regulated by bulb size in Lilium longiflorum (Easter lily). Plant Biol. 2016;18(4):577-584.

24. Li Y, Zhao Y, Zhang M, Jia G. Functional and evolutionary characterization of the CONSTANSlike family in Lilium $\times$ formolongi. Plant Cell Physiol. 2018;59(9):1874-1888.

25. Peruzzi L, Leitch IJ, Caparelli KF. Chromosome diversity and evolution in Liliaceae.Ann Bot. 2009;103(3):459-475.

26. Lin PC, Lu CW, Shen BN, Lee GZ, Bowman JL, Arteagavazquez MA, Liu LYD, Hong SF, Lo C, Su GM, et al. Identification of miRNAs and their targets in the liverwort Marchantia polymorpha by integrating RNA-Seq and degradome analyses. Plant Cell Physiol. 2016;57(2):339-358.

27. Edwards D, Batley J. Plant genome sequencing: applications for crop improvement. Plant Biotechnol J. 2010;8(1):2-9.

28. Yang X, Wang L, Yuan D, Lindsey K, Zhang X. Small RNA and degradome sequencing reveal complex miRNA regulation during cotton somatic embryogenesis. J Exp Bot. 2013;64:1521-1536.

29. Axtell MJ, Bartel DP. Antiquity of microRNAs and their targets in land plants. Plant Cell. 2005;17:1658-1673.

30. Xing L, Zhang D, Zhao C, Li Y, Ma J, An N, Han M. Shoot bending promotes flower bud formation by miRNA-mediated regulation in apple (Malus domestica). Plant Biotechnol J. 2016;14:749-770.

31. Yant L, Mathieu J, Dinh TT, Ott F, Lanz C, Wollmann H, Chen X, Schmid M. Orchestration of the floral transition and floral development in Arabidopsis by the bifunctional transcription factor APETALA2. Plant Cell. 2010;22:2156-2170.

32. Xie K, Shen J, Hou X, Yao J, Li X, Xiao J, Xiong L. Gradual increase of miR156 regulates temporal expression changes of numerous genes during leaf development in rice. Plant Physiol. 2012;158:1382-1394. 
33. Yang L, Conway SR, Poethig RS. Vegetative phase change is mediated by a leaf-derived signal that represses the transcription of miR156. Development. 2011;138:245-249.

34. Feng S, Xu Y, Guo C, Zheng J, Zhou B, Zhang Y, Ding Y, Zhang L, Zhu Z, Wang H, et al. Modulation of miR156 to identify traits associated with vegetative phase change in tobacco (Nicotiana tabacum). J Exp Bot. 2016;67:1493-1504.

35. Wang J, Park MY, Wang L, Koo Y, Chen X, Weigel D, Poethig RS. MiRNA control of vegetative phase change in trees. PLoS Genet. 2011;7:e1002012.

36. Yu S, Cao L, Zhou C, Zhang T, Lian H, Sun Y, Wu J, Huang J, Wang G, Wang J. Sugar is an endogenous cue for juvenile-to-adult phase transition in plants. eLife. 2013;2:e00269.

37. Francozorrilla JM, Valli A, Todesco M, Mateos I, Puga MI, Rubiosomoza I, Leyva A, Weigel D, Garcia JA, Pazares J. Target mimicry provides a new mechanism for regulation of microRNA activity. Nat Genet. 2007;39(8):1033-1037.

38. Xie K, Wu C, Xiong L. Genomic organization, differential expression, and interaction of SQUAMOSA promoter-binding-like transcription factors and microRNA156 in rice. Plant Physio. 2006;142(1):280-293.

39. Zhu Q, Helliwell CA. Regulation of flowering time and floral patterning by miR172. J Exp Bot. 2011;62(2):487-495.

40. Jung J, Seo Y, Seo PJ, Reyes JL, Yun J, Chua N, Park CM. The GIGANTEA-regulated microRNA172 mediates photoperiodic flowering independent of CONSTANS in Arabidopsis. Plant Cell. 2007;19(9):2736-2748.

41. Tang M, Bai X, Niu L, Chai X, Chen M, Xu Z. miR172 Regulates both vegetative and reproductive development in the perennial woody plant Jatropha curcas. Plant Cell Physiol. 2018;59:2549-2563.

42. Rubiosomoza I, Weigel D. Coordination of flower maturation by a regulatory circuit of three microRNAs. PLoS Genet. 2013;9(3):e1003374.

43. Tsuji H, Aya K, Ueguchitanaka M, Shimada Y, Nakazono M, Watanabe R, Nishizawa NK, Gomi K, Shimada A, Kitano H, et al. GAMYB controls different sets of genes and is differentially regulated by microRNA in aleurone cells and anthers. Plant J. 2006;47(3):427-444.

44. Achard P, Herr AJ, Baulcombe DC, Harberd NP. Modulation of floral development by a gibberellin-regulated microRNA. 2004;131(14):3357-3365.

45. Aceto S, Sica M, De Paolo S, D'Argenio V, Cantiello P, Salvatore F, Gaudio L. The analysis of the inflorescence miRNome of the orchid Orchis italica reveals a DEF-like MADS-box gene as a new miRNA target. PLoS ONE. 2014;9(5):e97839.

46. Preston JC, Hileman LC. Functional evolution in the plant SQUAMOSA-PROMOTER BINDING PROTEIN-LIKE (SPL) gene family. Front Plant Sci. 2013; 5(4):80-80.

47. Wagner D. Making flowers at the right time. Dev Cell. 2016;37(3):208-210.

48. Gou J, Tang C, Chen N, Wang H, Debnath S, Sun L, Flanagan A, Tang Y, Jiang Q, Allen RD, Wang Z. SPL7 and SPL8 represent a novel flowering regulation mechanism in switchgrass. New Phytol. 2019;222(3):1610-1623.

49. Alonsoperal MM, Li J, Li Y, Allen RS, Schnippenkoetter W, Ohms S, White RG, Millar AA. The microRNA159-regulated GAMYB-like genes inhibit growth and promote programmed cell death in Arabidopsis. Plant Physiol. 2010;154(2):757-771.

50. Lucidos JG, Ryu KB, Younis A, Kim C, Hwang Y, Son B, Lim K. Different day and night temperature responses in Lilium hansonii in relation to growth and flower development. Hortic Environ Biote. 2013;54(5):405-411.

51. Jeyaraj A, Zhang X, Hou Y, Shangguan M, Gajjeraman P, Li Y, Wei C. Genome-wide identification of conserved and novel microRNAs in one bud and two tender leaves of tea plant (Camellia sinensis) by small RNA sequencing, microarray-based hybridization and genome survey scaffold sequences. BMC Plant Biol. 2017;17(1):212-212..

52. Li X, Shahid MQ, Wu J, Wang L, Liu X, Lu Y. Comparative small RNA analysis of pollen development in autotetraploid and diploid rice. Int J Mol Sc. 2016;17(4):499-499.

53. Addo-Quaye C, Eshoo TW, Bartel DP, Axtell MJ. Endogenous siRNA and miRNA targets identified by sequencing of the Arabidopsis Curr Biol. 2008;18(10):758-762.

54. Jonesrhoades MW, Bartel DP. Computational identification of plant microRNAs and their targets, including a stress-induced miRNA. Mol Cell. 2004;14(6):787-799.

55. Folkes L, Moxon S, Woolfenden HC, Stocks MB, Szittya G, Dalmay T, Moulton V. PAREsnip: a tool for rapid genome-wide discovery of small RNA/target interactions evidenced through degradome sequencing. Nucleic Acids Res. 2012;40(13):e103.

56. Xie F, Frazier TP, Zhang B. Identification and characterization of microRNAs and their targets in the bioenergy plant switchgrass (Panicum virgatum). Planta. 2010;232:417-434.

57. Livak KJ, Schmittgen TD. Analysis of relative gene expression data using real-time quantitative PCR and the 2 (-Delta Delta C (T)) method. Methods. 2001;25(4):402-408.

\section{Figures}



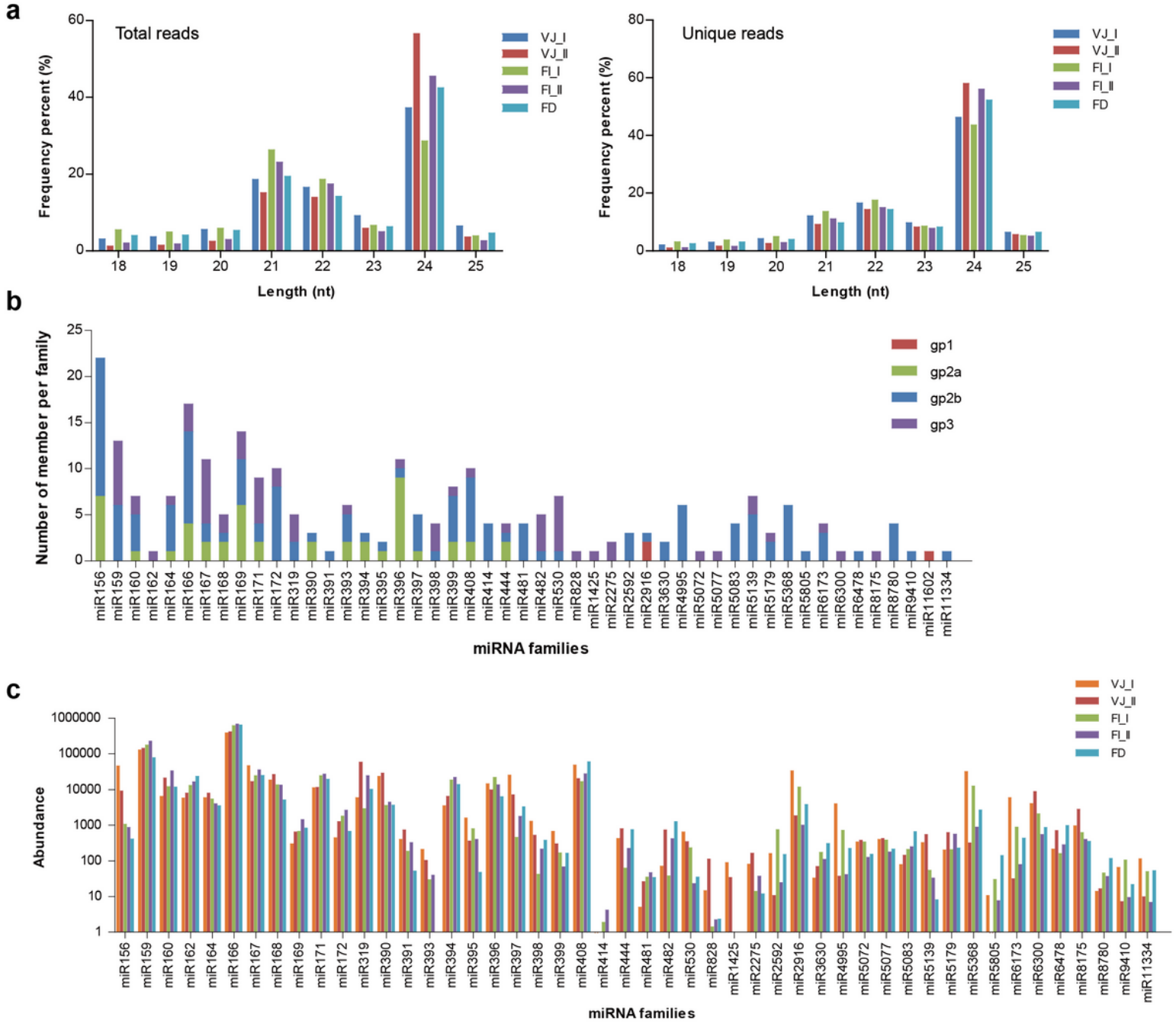

\section{Figure 1}

Length distribution of small RNAs sequences, identification of known miRNA families from L. $\times$ formolongi. a Length distribution of total reads and unique reads in five libraries. b Distribution of known miRNA family members. c Counts of each known miRNA family 
a

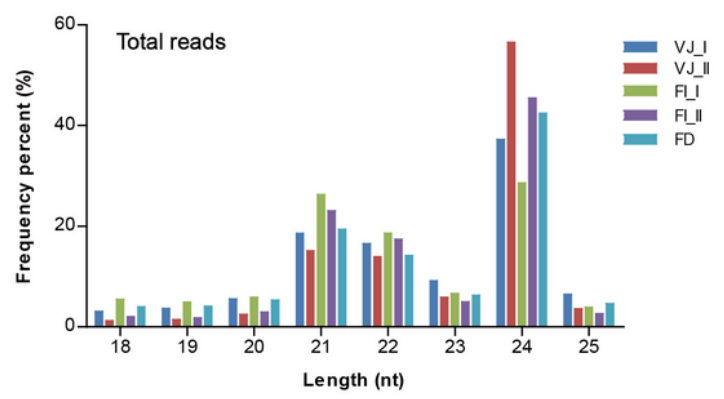

b
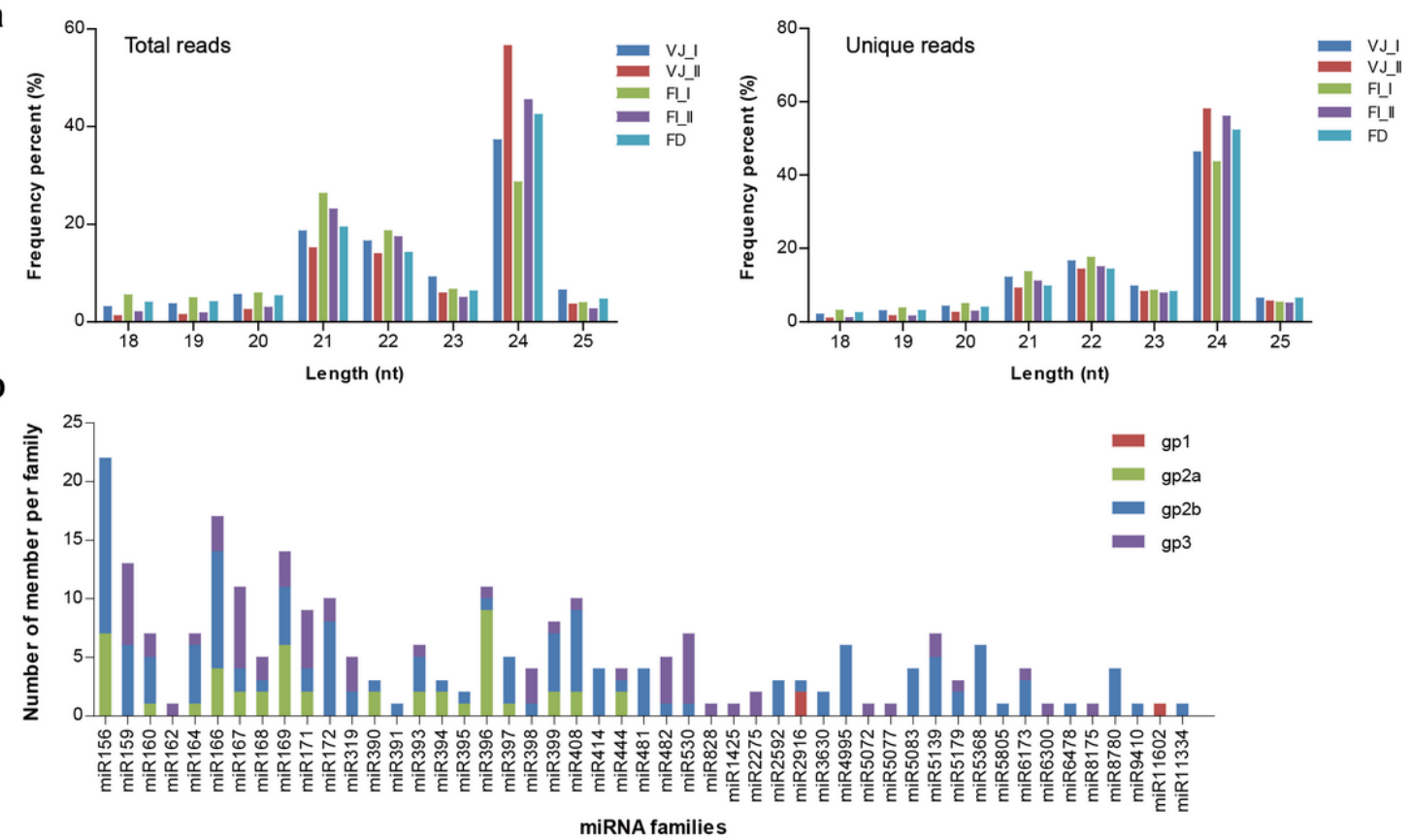

C

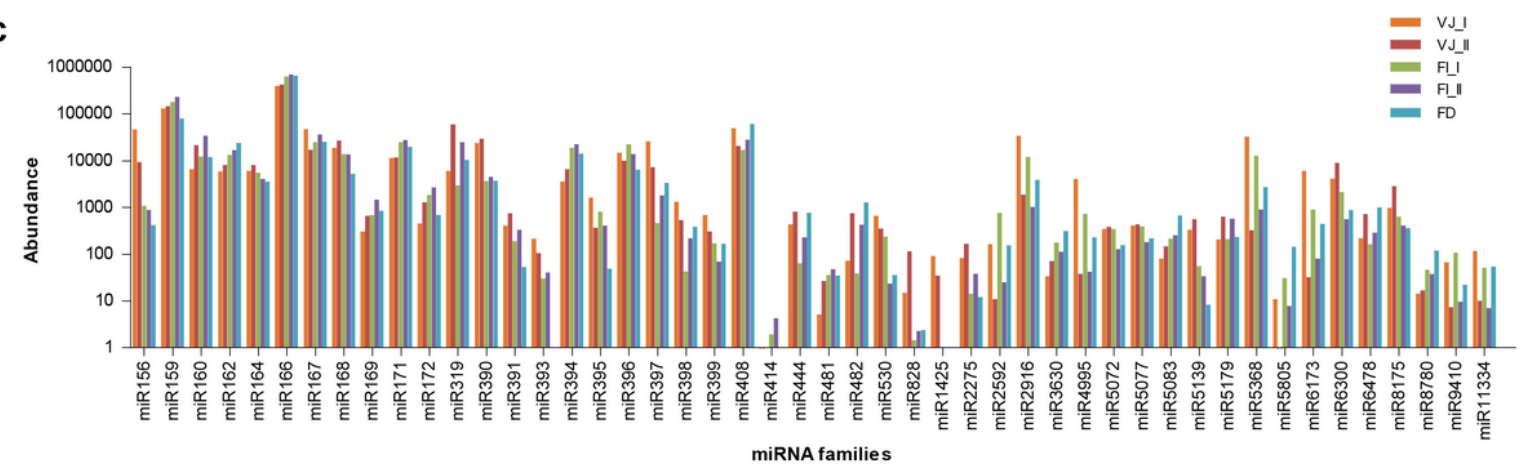

Figure 1

Length distribution of small RNAs sequences, identification of known miRNA families from L. $\times$ formolongi. a Length distribution of total reads and unique reads in five libraries. b Distribution of known miRNA family members. c Counts of each known miRNA family 


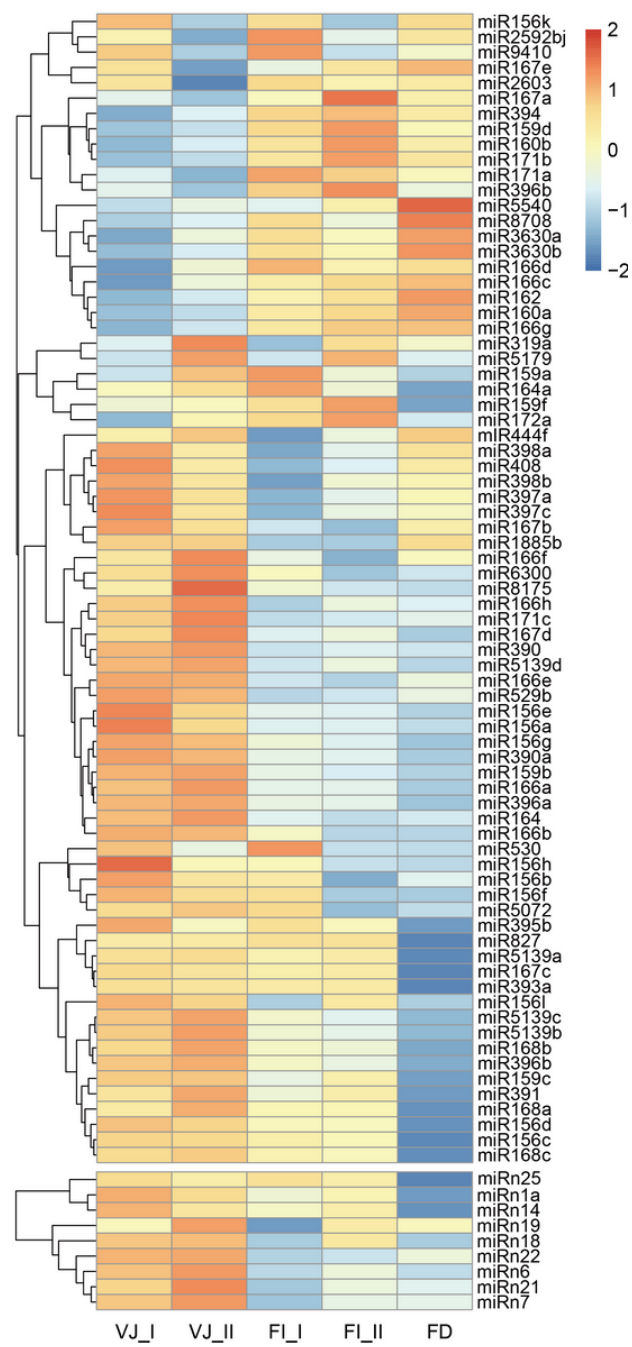

\section{Figure 2}

Heat map showing the expression patterns of differentially expressed miRNAs. The miRNAs were clustered by hierarchical clustering at $\mathrm{p} \leq 0.05$ according to their expression patterns during development in L.x formolongi 


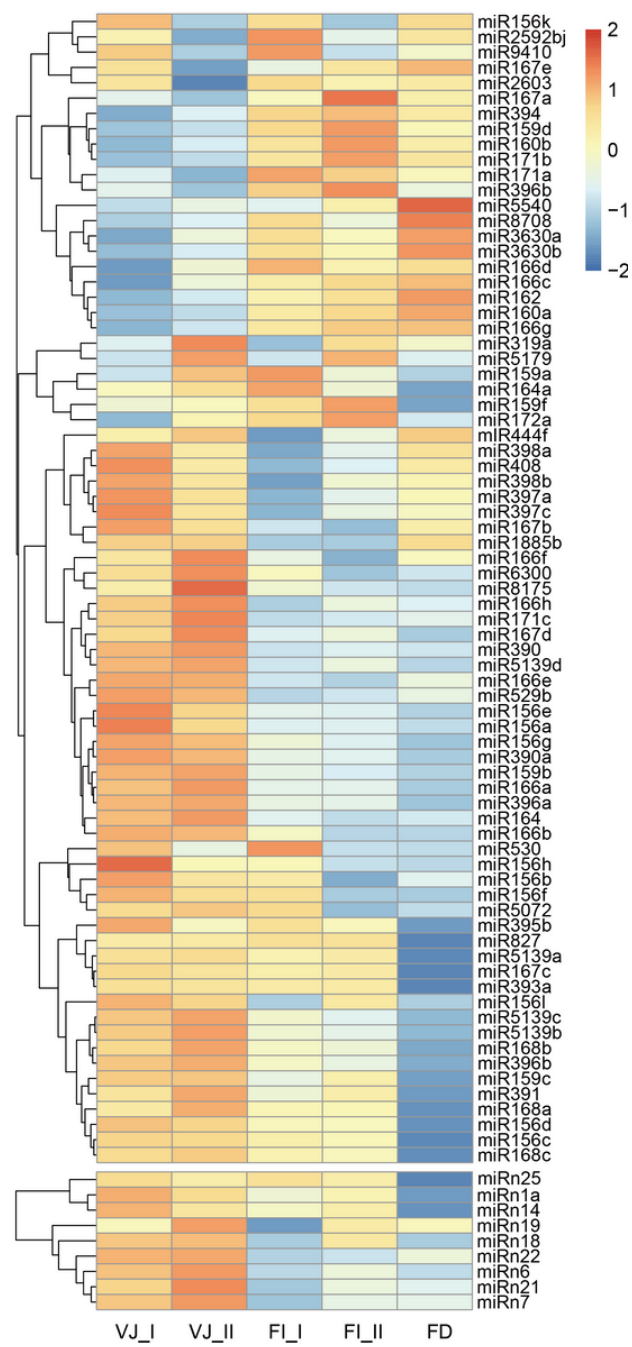

\section{Figure 2}

Heat map showing the expression patterns of differentially expressed miRNAs. The miRNAs were clustered by hierarchical clustering at $\mathrm{p} \leq 0.05$ according to their expression patterns during development in L.x formolongi 
a

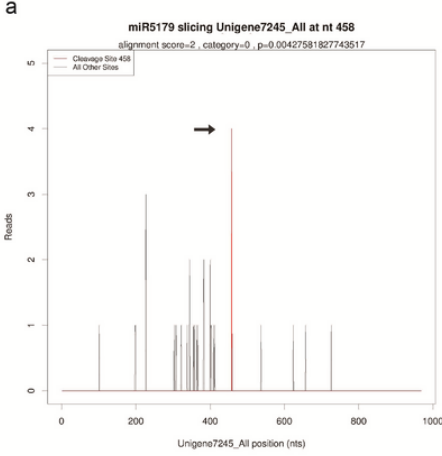

Unigene7245_All LAGL15

$\downarrow$

5' - GACCUGCGCGGUCuUGAGCAAAA - $3^{\prime}$

$3^{\prime}$ - ACCAACGCGCCAGAACUCGUUUU -5'

miR5179

c

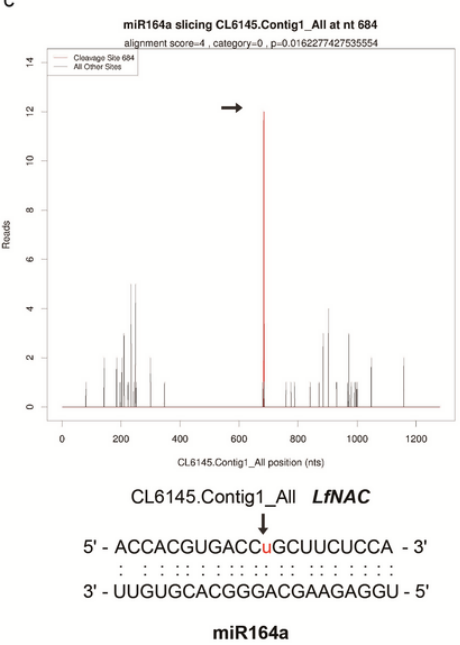

b

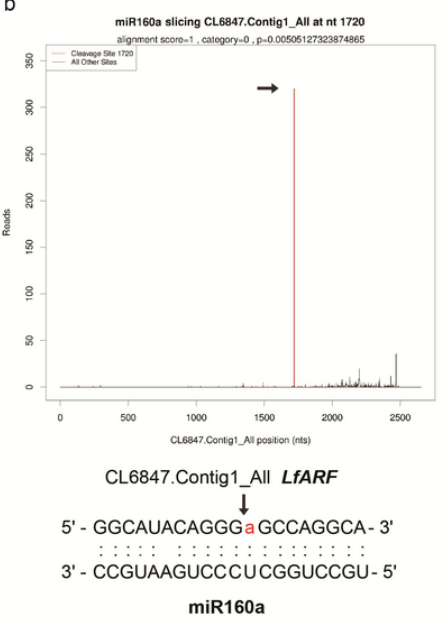

d

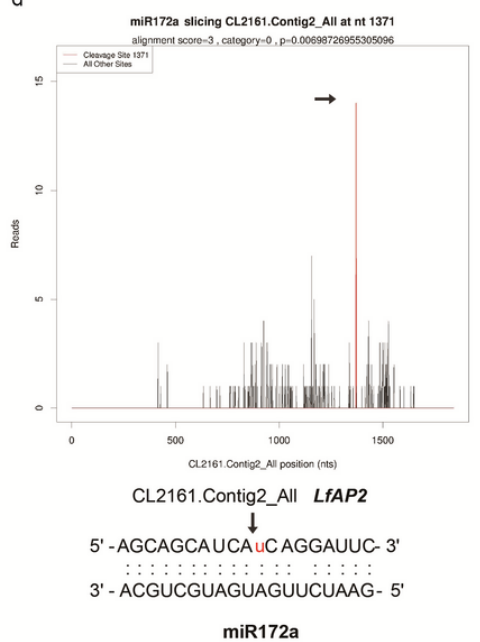

\section{Figure 3}

Target plots of miRNA targets identified by degradome sequencing in lily a-d represent miR5179, miR160a, miR164a and miR172a, respectively. Arrows represent the nucleotide position of cleavage in the target genes. 
a

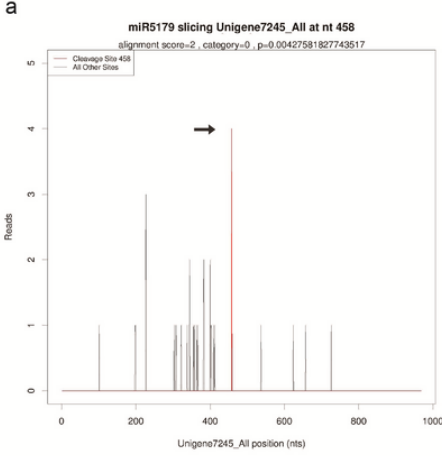

Unigene7245_All LAGL15

$\downarrow$

5' - GACCUGCGCGGUCuUGAGCAAAA - $3^{\prime}$

$3^{\prime}$ - ACCAACGCGCCAGAACUCGUUUU -5'

miR5179

c

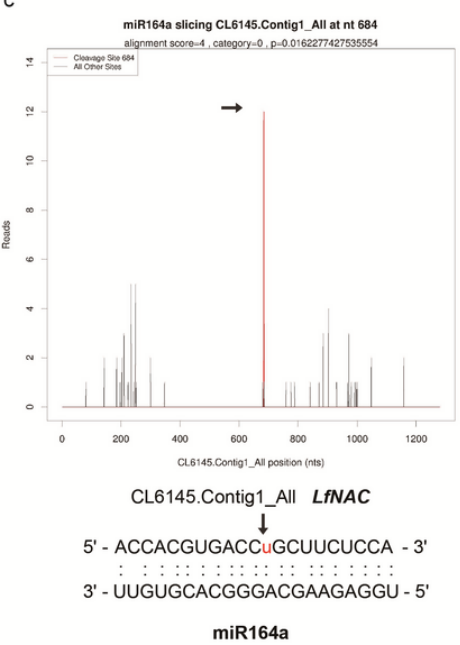

b

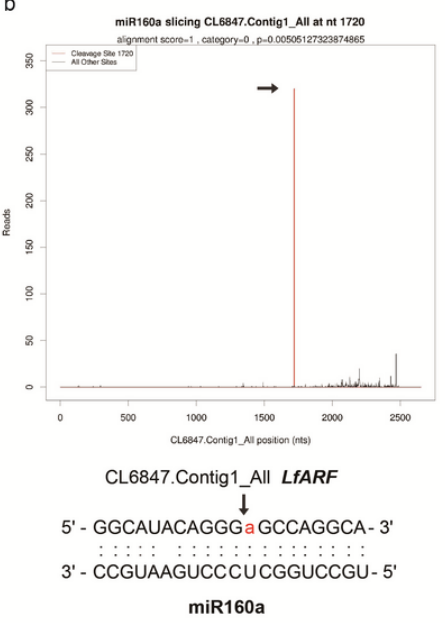

d

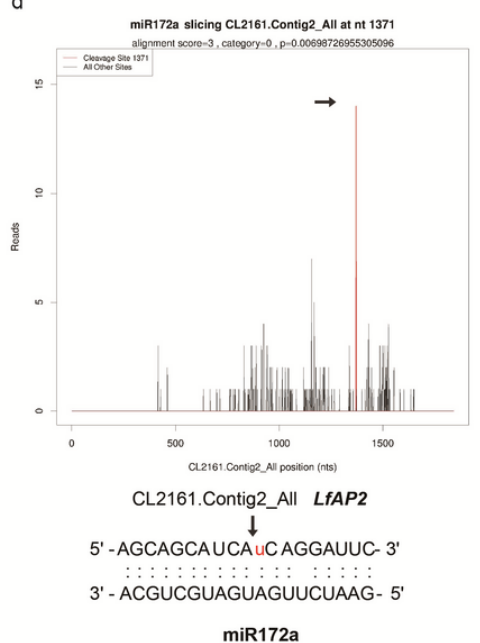

\section{Figure 3}

Target plots of miRNA targets identified by degradome sequencing in lily a-d represent miR5179, miR160a, miR164a and miR172a, respectively. Arrows represent the nucleotide position of cleavage in the target genes. 
a

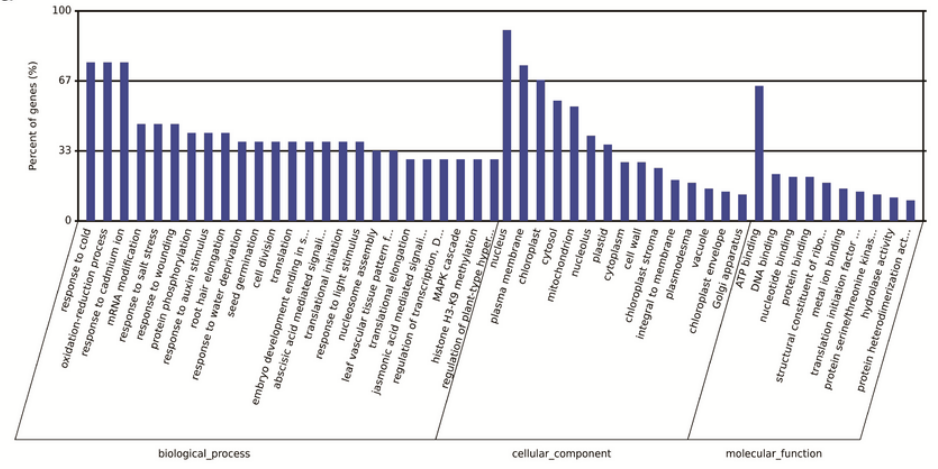

b

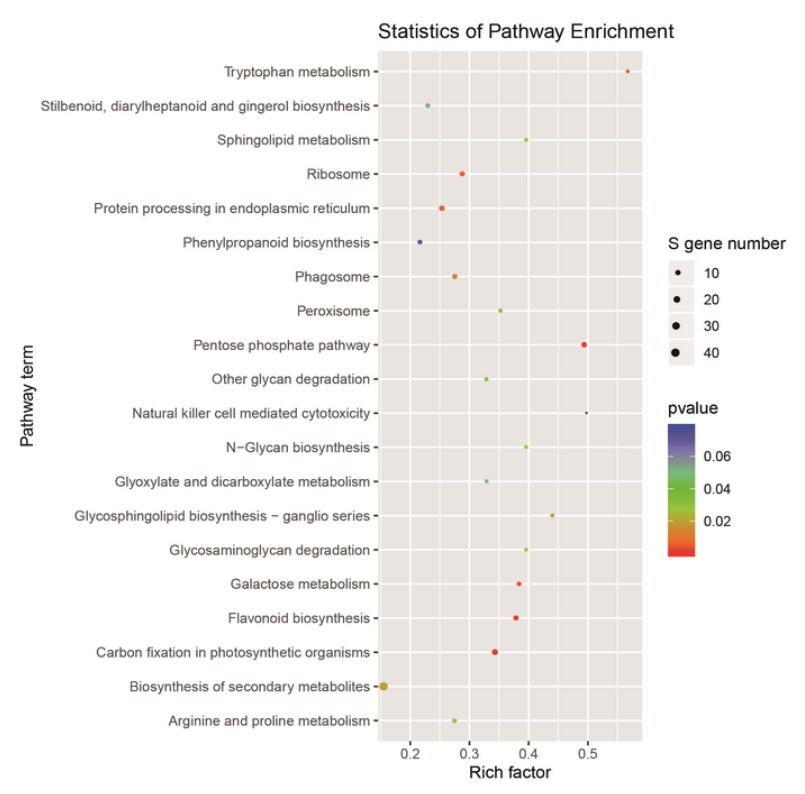

Figure 4

Gene functional classification of identified target genes. a Gene ontology enrichment and classification of target genes. b KEGG pathway analysis 
a

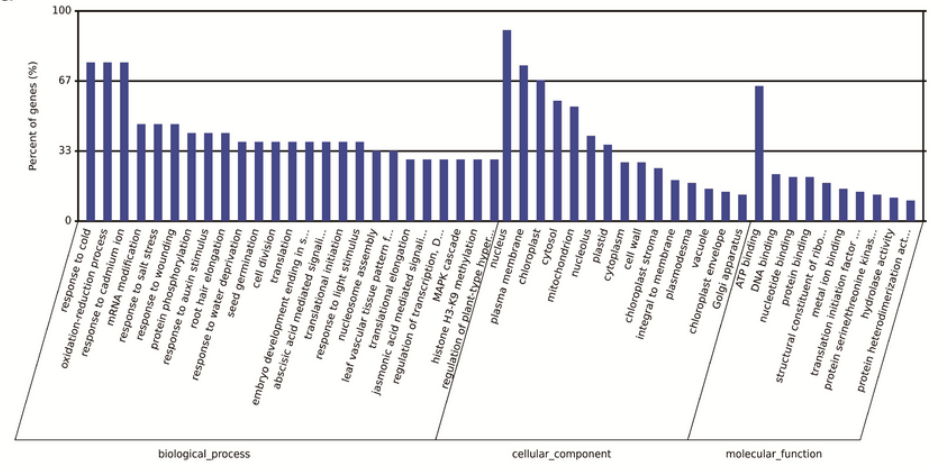

b

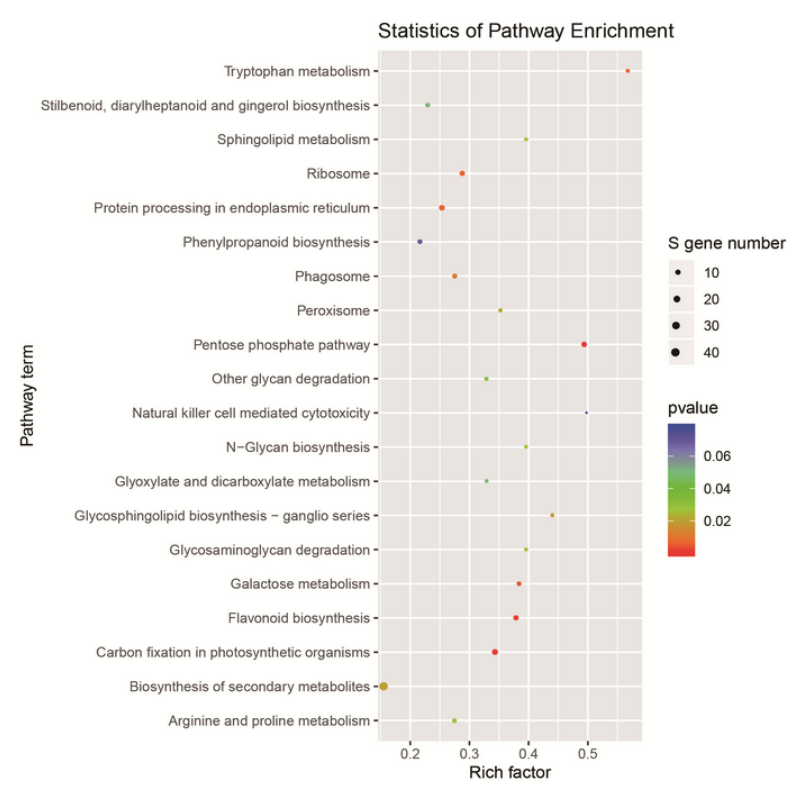

Figure 4

Gene functional classification of identified target genes. a Gene ontology enrichment and classification of target genes. b KEGG pathway analysis 

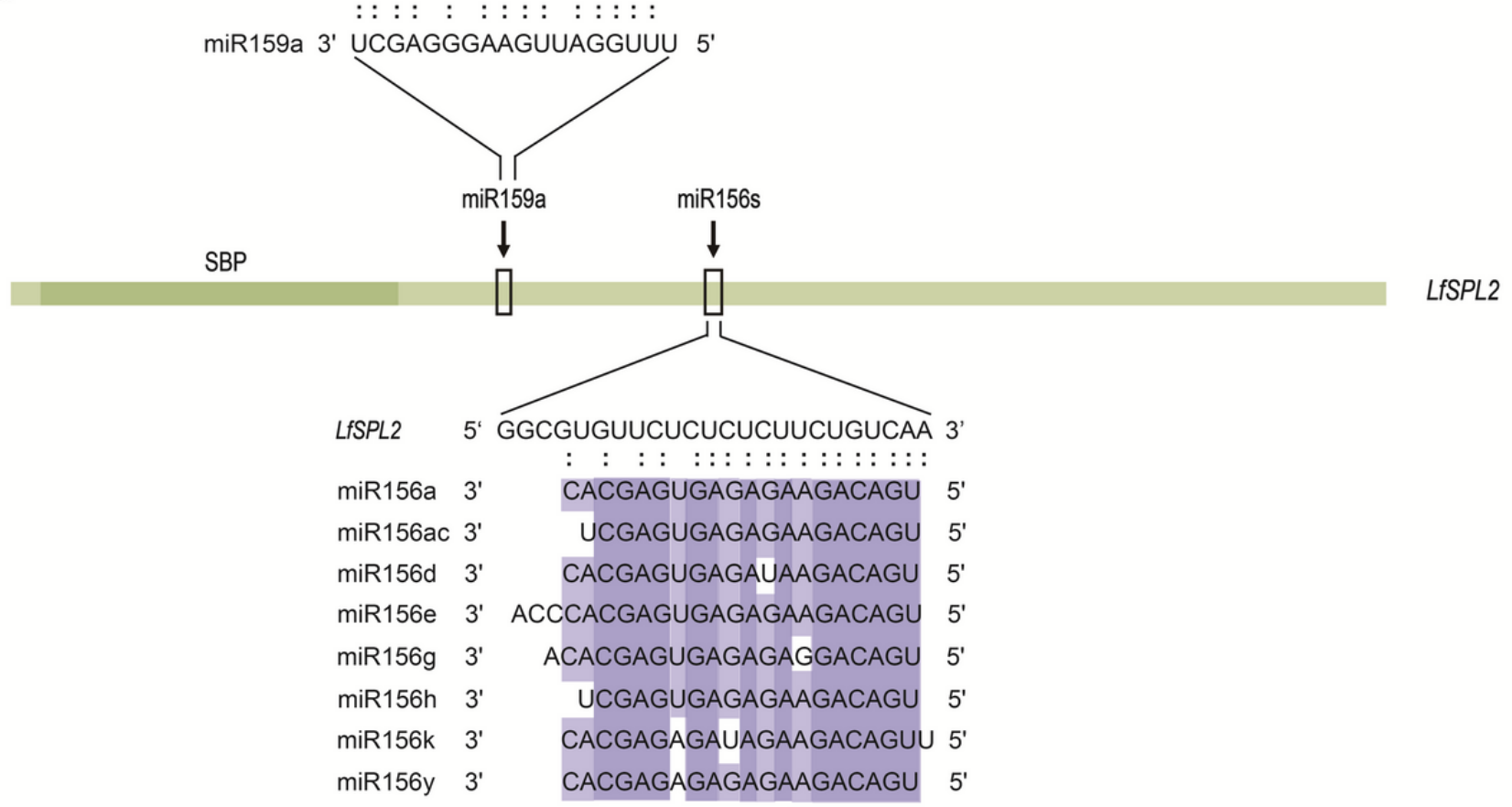

b

PLN03212

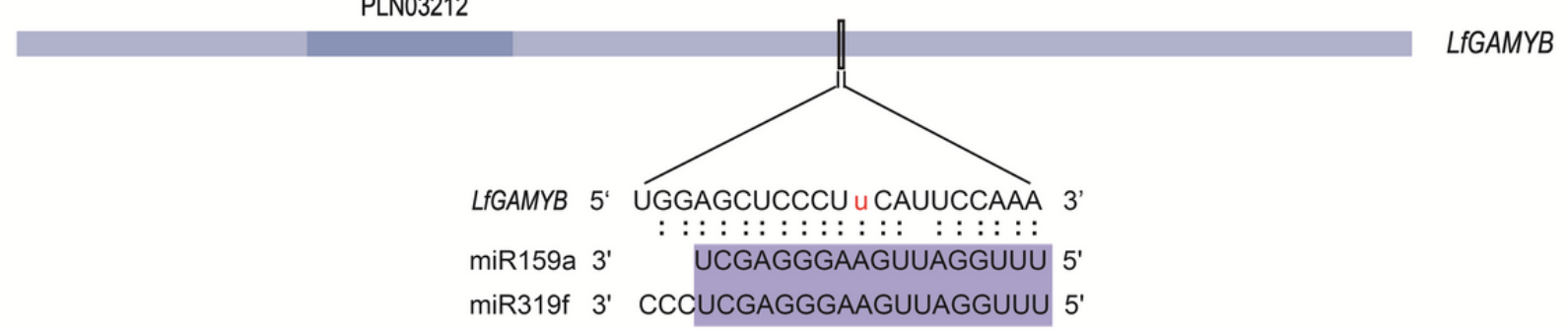

Figure 5

mRNAs simultaneously targeted by miRNAs belonging to multiple families. a Diagram of LfSPL2 and LfGAMYB. Conserved domains are included, and the boxes represent the miRNA target sites. b Alignment of the miRNA targeting sites of miR156s-LfSPL2, miR159a-LfSPL2 and miR159a/miR319f-LfGAMYB 

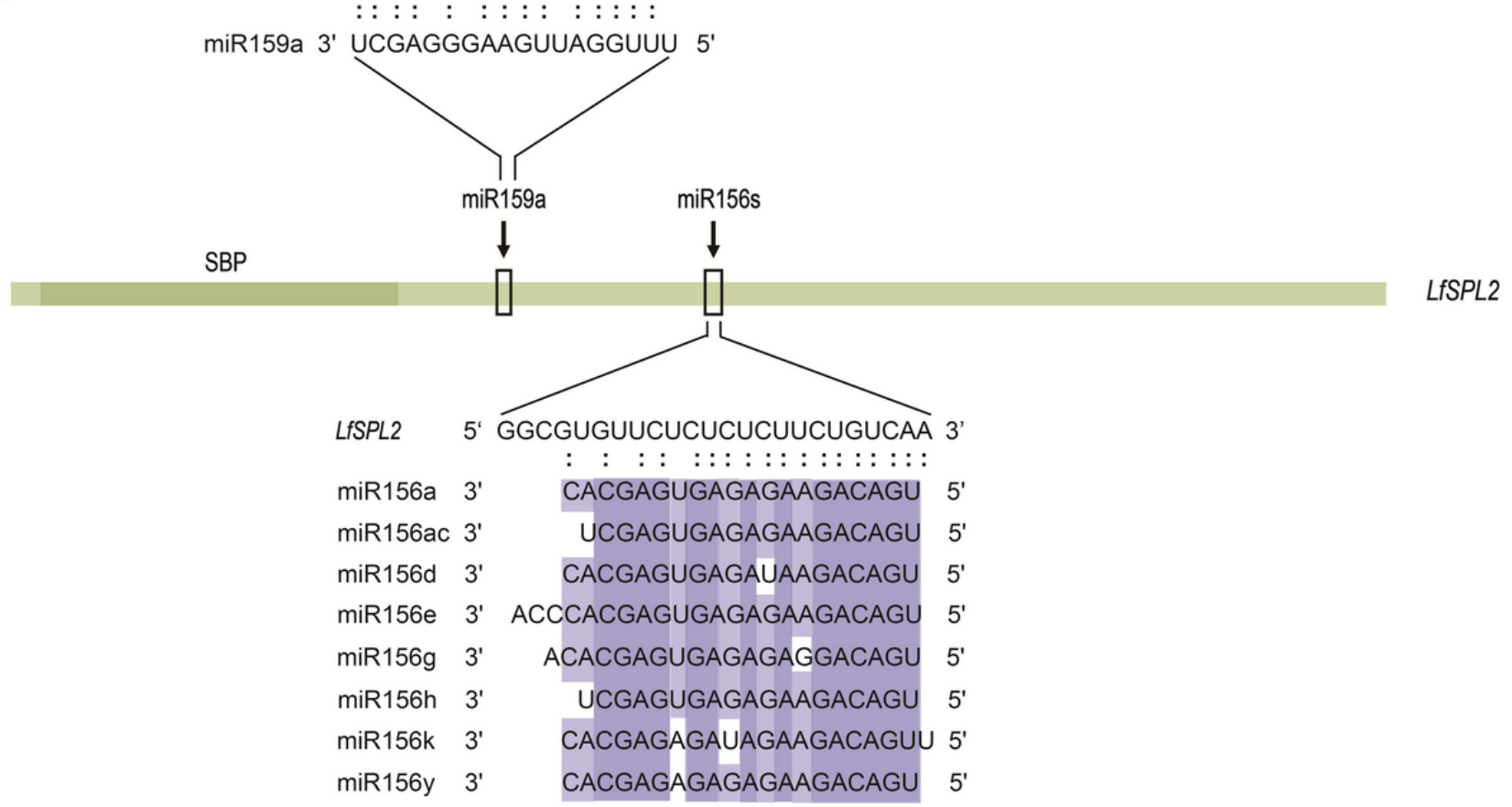

b

PLN03212

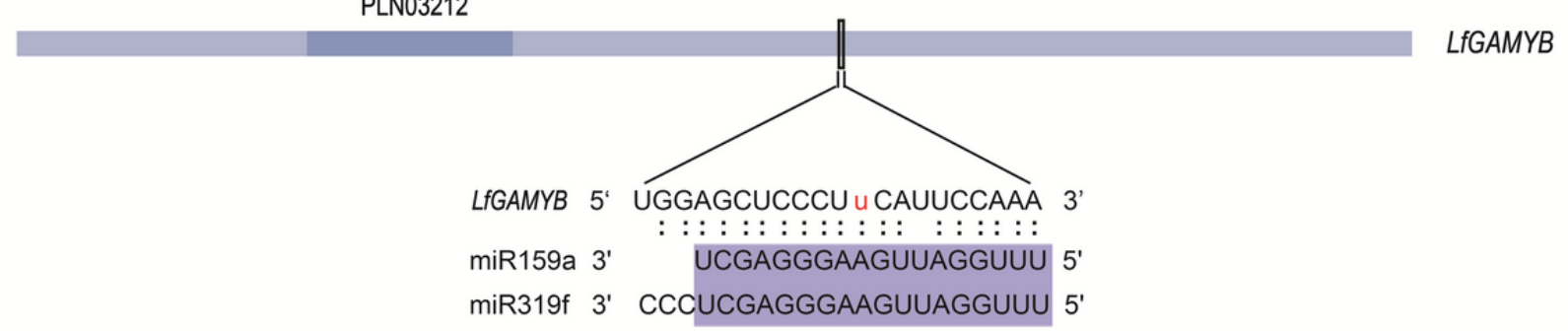

Figure 5

mRNAs simultaneously targeted by miRNAs belonging to multiple families. a Diagram of LfSPL2 and LfGAMYB. Conserved domains are included, and the boxes represent the miRNA target sites. b Alignment of the miRNA targeting sites of miR156s-LfSPL2, miR159a-LfSPL2 and miR159a/miR319f-LfGAMYB 


\begin{tabular}{|c|c|c|c|}
\hline & & & miR398a \\
\hline & & & miR397a \\
\hline & & .85 & miR156y \\
\hline & & 0.66 & miR156a \\
\hline & & 0.77 & miR156e \\
\hline & & -0.78 & miR156h \\
\hline & & -0.96 & miR156g \\
\hline & & -0.85 & miR169b \\
\hline & & -1.39 & miR156d \\
\hline & & -1.48 & miR156ac \\
\hline & & 1.15 & miR8708 \\
\hline & & 0.13 & miR2592bj \\
\hline & & 0.03 & miR7825 \\
\hline & & -0.19 & miR169c \\
\hline & & -0.76 & miR396b \\
\hline & & -0.76 & miR396f \\
\hline & & -0.76 & miR396f \\
\hline & & -0.76 & miR396b \\
\hline & & -0.59 & miR172a \\
\hline & & -0.59 & miR172a \\
\hline & & -0.59 & miR172a \\
\hline & .05 & -0.78 & miR159a \\
\hline & .09 & -1.24 & miR164a \\
\hline FI_I & FI_II & FD & \\
\hline
\end{tabular}

\begin{tabular}{|c|c|c|c|c|}
\hline 1.28 & -0.08 & -0.02 & -1.17 & Unigene13871_All LfCSD \\
\hline 1.40 & -0.98 & -0.18 & -0.24 & CL503.Contig1_Al LfLACs \\
\hline-1.44 & 0.69 & 0.10 & 0.65 & Unigene18229_All LfSPL2 \\
\hline-1.44 & 0.69 & 0.10 & 0.65 & Unigene18229_All LfSPL2 \\
\hline-1.44 & 0.69 & 0.10 & 0.65 & Unigene18229_All LfSPL2 \\
\hline-1.44 & 0.69 & 0.10 & 0.65 & Unigene18229_All LfSPL2 \\
\hline-1.44 & 0.69 & 0.10 & 0.65 & Unigene18229_All LfSPL2 \\
\hline-1.39 & 0.97 & 0.08 & 0.34 & Unigene9425_All Unknown \\
\hline-1.44 & 0.69 & 0.10 & 0.65 & Unigene18229_All LfSPL2 \\
\hline-1.44 & 0.69 & 0.10 & 0.65 & Unigene18229_All LfSPL2 \\
\hline-1.43 & 0.92 & 0.28 & 0.23 & Unigene8156_All LfFAR3 \\
\hline-0.53 & -1.01 & 1.27 & 0.28 & CL171.Contig2_All LfnlpC \\
\hline 1.28 & -1.16 & -0.15 & 0.02 & Unigene901_All hypothetical protein \\
\hline-1.43 & 0.18 & 0.36 & 0.89 & CL807.Contig2_All hypothetical protein \\
\hline-1.28 & 1.07 & -0.21 & 0.41 & Unigene4858_All hypothetical protein \\
\hline-1.28 & 1.07 & -0.21 & 0.41 & Unigene4858_All hypothetical protein \\
\hline-1.49 & 0.58 & 0.31 & 0.60 & Unigene14983_All Unknown \\
\hline-1.49 & 0.58 & 0.31 & 0.60 & Unigene14983_All Unknown \\
\hline 0.52 & 1.06 & -1.21 & -0.37 & Unigene12377_All Unknown \\
\hline 0.19 & 1.34 & -0.71 & -0.82 & CL2161.Contig3_All Unknown \\
\hline 0.23 & 1.29 & -0.52 & -1.00 & Unigene18548_All LfAP2 \\
\hline-1.44 & 0.69 & 0.10 & 0.65 & Unigene18229_All LfSPL2 \\
\hline 0.48 & 1.06 & -1.26 & -0.28 & CL6145.Contig1_All LfNAC \\
\hline VJ & FI_I & FI_II & FD & \\
\hline
\end{tabular}

Figure 6

Heat map showing the expression patterns of differentially expressed miRNA-target. These miRNAs targeting differentially expressed mRNAs were clustered by hierarchical clustering at $\mathrm{p} \leq 0.05$ according to their expression patterns

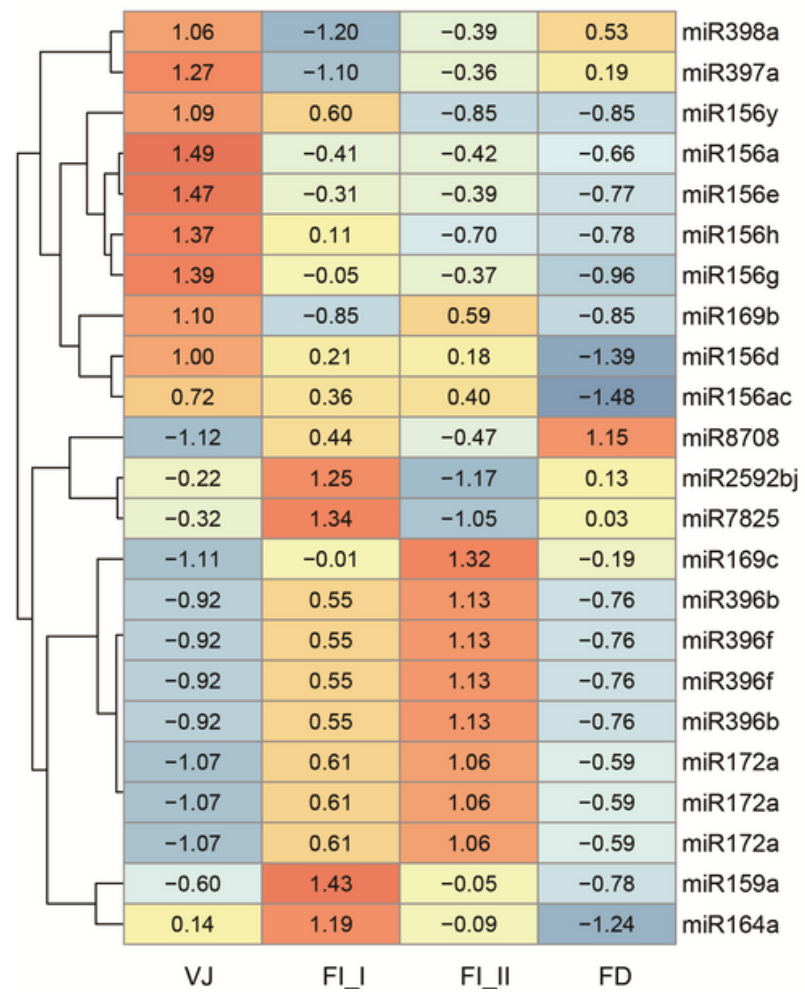

\begin{tabular}{|c|c|c|c|l|l}
\hline 1.28 & -0.08 & -0.02 & -1.17 & Unigene13871_All LfCSD \\
\hline 1.40 & -0.98 & -0.18 & -0.24 & CL503.Contig1_Al LfLACs \\
-1.44 & 0.69 & 0.10 & 0.65 & Unigene18229_All LfSPL2 \\
\hline-1.44 & 0.69 & 0.10 & 0.65 & Unigene18229_All LfSPL2 \\
-1.44 & 0.69 & 0.10 & 0.65 & Unigene18229_All LfSPL2 \\
-1.44 & 0.69 & 0.10 & 0.65 & Unigene18229_All LfSPL2 \\
-1.44 & 0.69 & 0.10 & 0.65 & Unigene18229_All LfSPL2 \\
\hline-1.39 & 0.97 & 0.08 & 0.34 & Unigene9425_All Unknown \\
\hline-1.44 & 0.69 & 0.10 & 0.65 & Unigene18229_All LfSPL2 \\
\hline-1.44 & 0.69 & 0.10 & 0.65 & Unigene18229_All LfSPL2 \\
\hline-1.43 & 0.92 & 0.28 & 0.23 & Unigene8156_All LfFAR3 \\
\hline-0.53 & -1.01 & 1.27 & 0.28 & CL171.Contig2_All LfnlpC \\
\hline 1.28 & -1.16 & -0.15 & 0.02 & Unigene901_All hypothetical protein \\
\hline-1.43 & 0.18 & 0.36 & 0.89 & CL807.Contig2_All hypothetical protein \\
\hline-1.28 & 1.07 & -0.21 & 0.41 & Unigene4858_All hypothetical protein \\
\hline-1.28 & 1.07 & -0.21 & 0.41 & Unigene4858_All hypothetical protein \\
\hline-1.49 & 0.58 & 0.31 & 0.60 & Unigene14983_All Unknown \\
\hline-1.49 & 0.58 & 0.31 & 0.60 & Unigene14983_All Unknown \\
\hline 0.52 & 1.06 & -1.21 & -0.37 & Unigene12377_All Unknown \\
\hline 0.19 & 1.34 & -0.71 & -0.82 & CL2161.Contig3_All Unknown \\
\hline 0.23 & 1.29 & -0.52 & -1.00 & Unigene18548_All LfAP2 \\
\hline-1.44 & 0.69 & 0.10 & 0.65 & Unigene18229_All LfSPL2 \\
\hline 0.48 & 1.06 & -1.26 & -0.28 & CL6145.Contig1_All LfNAC \\
\hline VJ & Fl_I & FI_ll & FD & \\
\hline & & & &
\end{tabular}

Figure 6

Heat map showing the expression patterns of differentially expressed miRNA-target. These miRNAs targeting differentially expressed mRNAs were clustered by hierarchical clustering at $\mathrm{p} \leq 0.05$ according to their expression patterns 


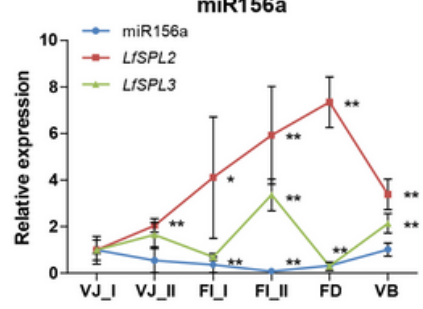

e
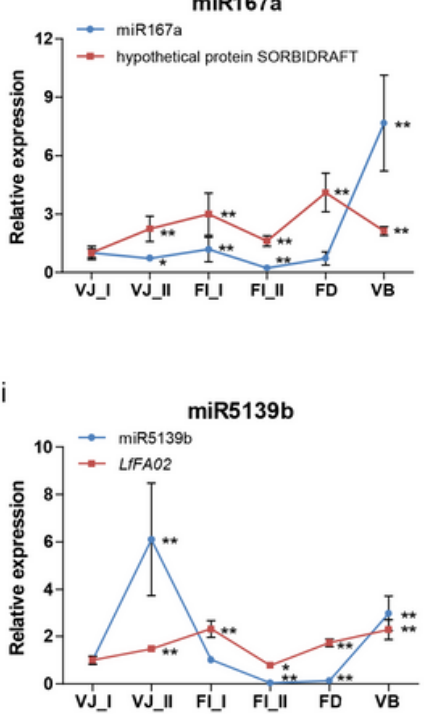

b

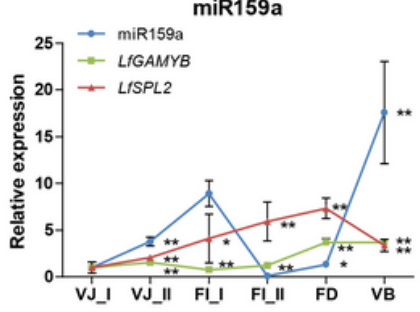

f

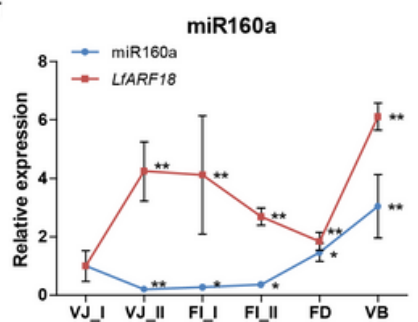

j

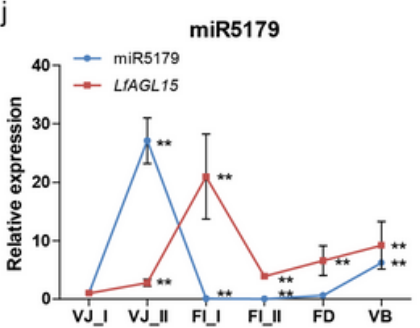

C

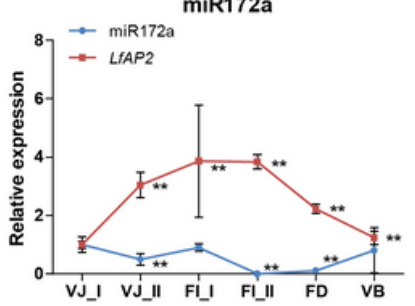

g

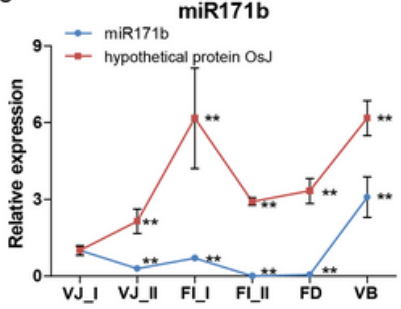

k

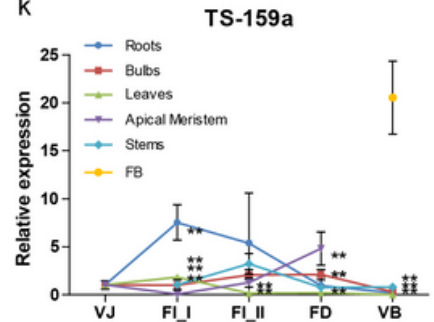

d

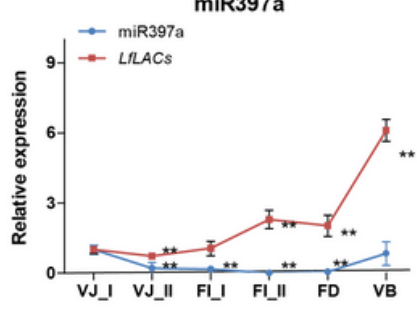

h

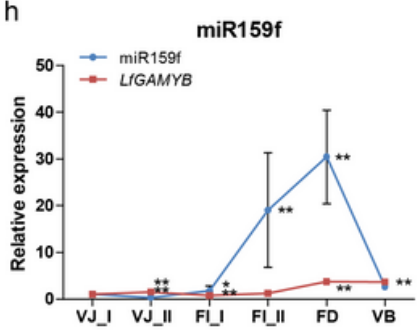

I

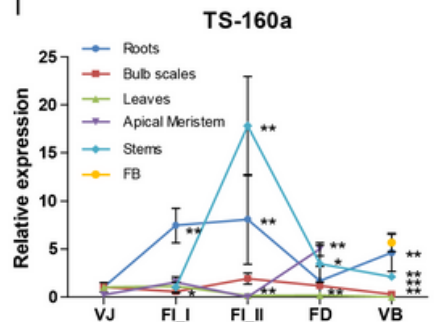

Figure 7

The expression patterns of miRNAs determined by qRT-PCR during development in L. $\times$ formolongi. a-j The expression patterns of miRNAs and their targets. $\mathrm{k}-\mathrm{I}$ The tissue-specific expression profiles of miR159a and miR160a during lily development processes. The normalized miRNA and target levels at the VJ_I stage were arbitrarily set to 1 . Each bar represents the mean \pm SE of triplicate assays. * or ** indicates a statistically significant difference as relative to the value at VJ_ I for each miRNAs at $p<0.05$ or 0.01 , respectively 


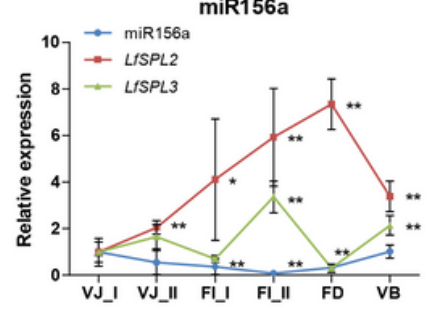

e

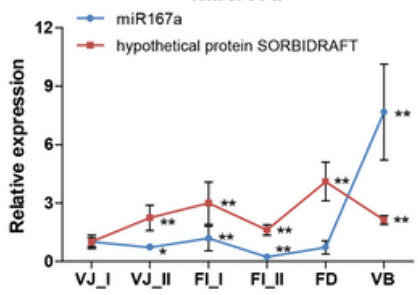

i

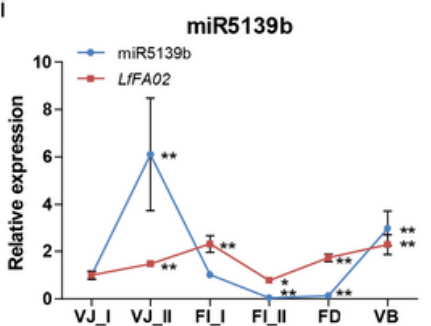

b

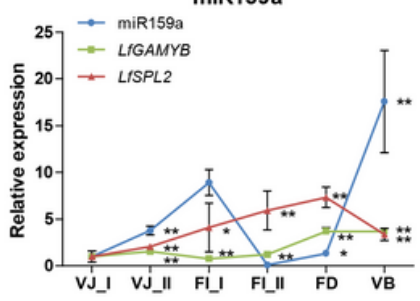

f

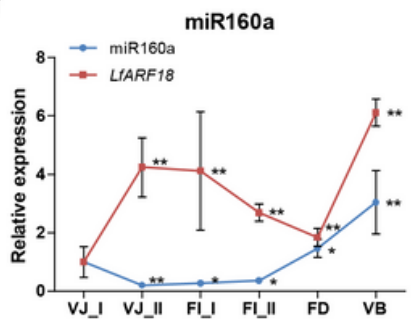

j

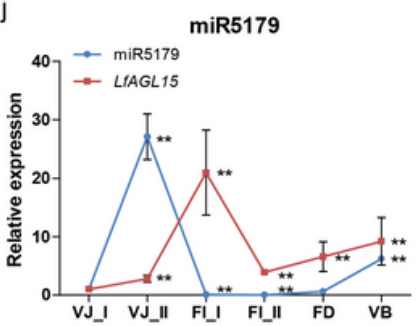

C

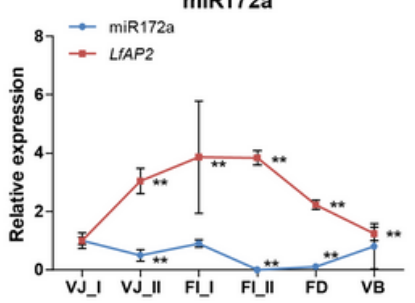

g

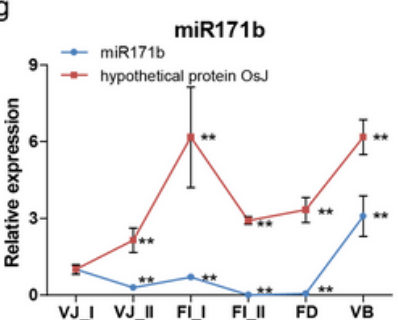

k

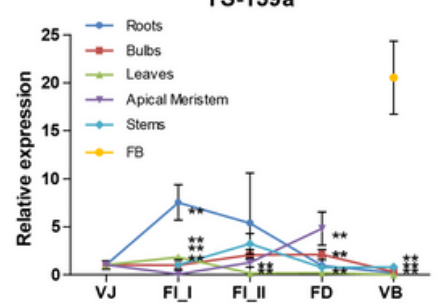

d

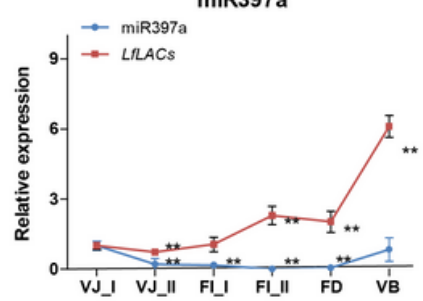

h

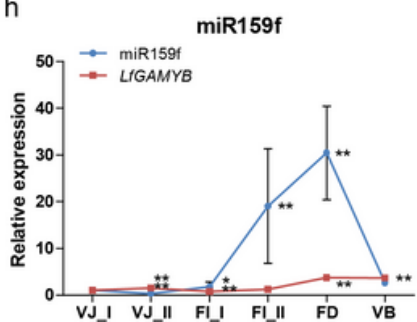

I

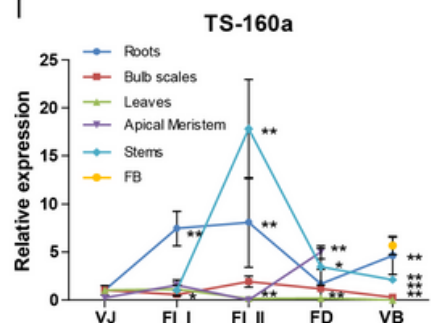

Figure 7

The expression patterns of miRNAs determined by qRT-PCR during development in L. $\times$ formolongi. a-j The expression patterns of miRNAs and their targets. $\mathrm{k}-\mathrm{I}$ The tissue-specific expression profiles of miR159a and miR160a during lily development processes. The normalized miRNA and target levels at the VJ_I stage were arbitrarily set to 1 . Each bar represents the mean \pm SE of triplicate assays. * or ** indicates a statistically significant difference as relative to the value at VJ_I for each miRNAs at $p<0.05$ or 0.01 , respectively

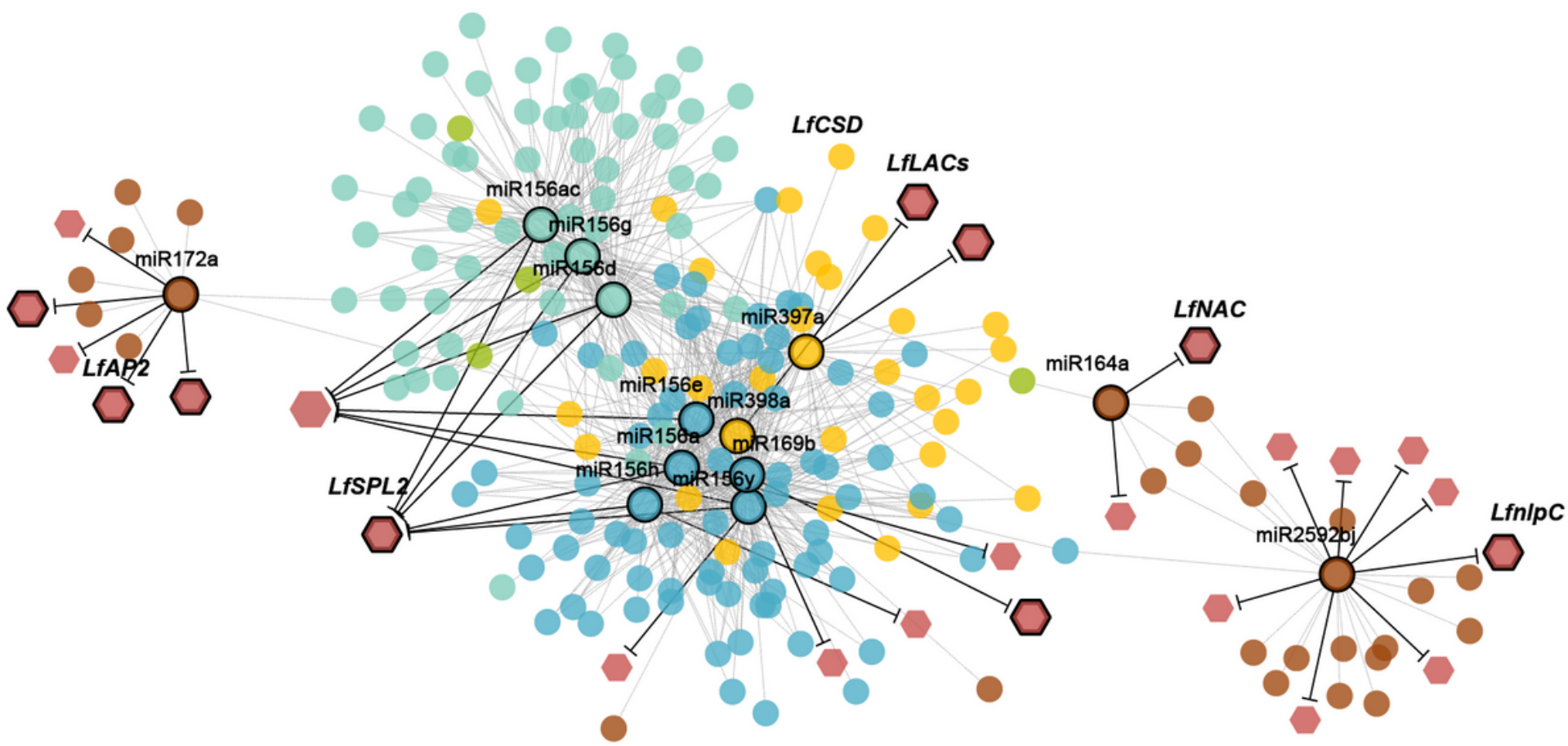

Figure 8 
The coexpression subnetwork of putative crucial flowering-related miRNAs and their targets. The coexpression subnetwork of putative crucial flowering-related miRNAs and their targets in lily. In the nodes, circles and diamonds represent miRNAs and target transcripts, respectively. Different colours of nodes represent different modules identified by WGCNA.

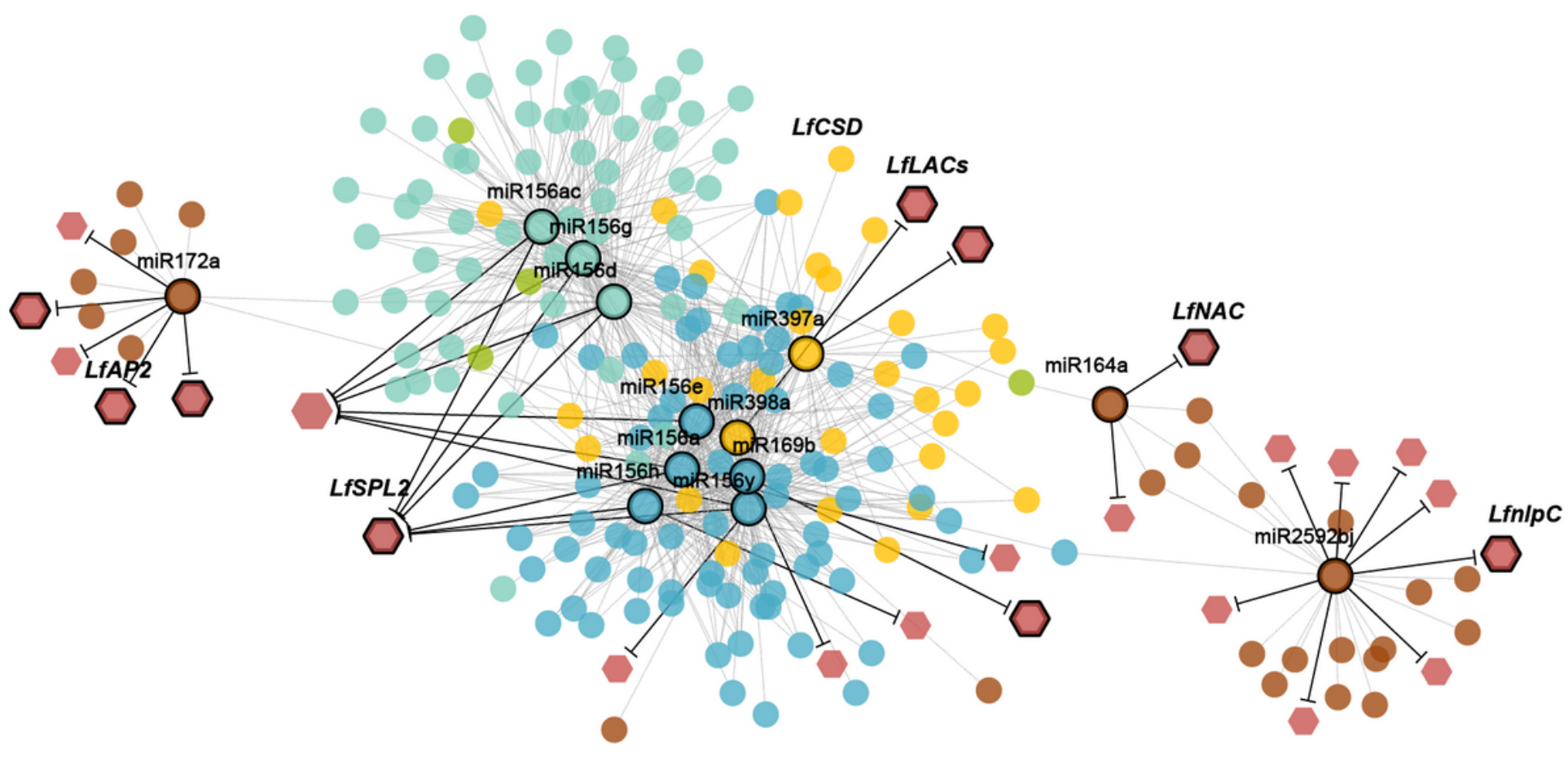

Figure 8

The coexpression subnetwork of putative crucial flowering-related miRNAs and their targets. The coexpression subnetwork of putative crucial flowering-related miRNAs and their targets in lily. In the nodes, circles and diamonds represent miRNAs and target transcripts, respectively. Different colours of nodes represent different modules identified by WGCNA.

\section{Supplementary Files}

This is a list of supplementary files associated with this preprint. Click to download.

- Additionalfile1.xlsx

- Additionalfile1.xlsx

- Fig.S1.tif

- Fig.S1.tif

- Fig.S2.emf

- Fig.S2.emf

- Additionalfile4.docx

- Additionalfile4.docx 